\title{
An Integrative Literature Review on the Nomenclature and Definition of Dementia at a Young Age
}

\author{
Dennis van de Veen ${ }^{\mathrm{a}, \mathrm{b}, \mathrm{c}}$, Christian Bakker ${ }^{\mathrm{a}, \mathrm{b}, \mathrm{d}, *}$, Kirsten Peetoom $^{\mathrm{e}}$, Yolande Pijnenburg ${ }^{\mathrm{f}}$, \\ Janne Papma ${ }^{\mathrm{g}}$, The PRECODE Study Group, Marjolein de Vugt ${ }^{\mathrm{e}}$ and Raymond Koopmans ${ }^{\mathrm{a}, \mathrm{b}, \mathrm{h}}$ \\ ${ }^{a}$ Department of Primary and Community Care, Radboud University Medical Center, Nijmegen, the Netherlands \\ ${ }^{\mathrm{b}}$ Radboudumc Alzheimer Center, Nijmegen, the Netherlands \\ ${ }^{\mathrm{c}}$ Florence, Mariahoeve, Center for Specialized Care in Young-Onset Dementia, The Hague, the Netherlands \\ ${ }^{\mathrm{d}}$ Groenhuysen, Center for Specialized Geriatric Care, Roosendaal, the Netherlands \\ e Alzheimer Center Limburg, Maastricht University, Maastricht, the Netherlands \\ ${ }_{\mathrm{f}}^{\mathrm{f}}$ Alzheimer Center Amsterdam, Department of Neurology, Amsterdam Neuroscience, Vrije Universiteit \\ Amsterdam, Amsterdam University Medical Center, Amsterdam, the Netherlands \\ ${ }^{\mathrm{g}}$ Department of Neurology and Alzheimer Center, Erasmus Medical Center Rotterdam, Rotterdam, \\ the Netherlands \\ ${ }^{\mathrm{h}}$ Joachim en Anna, Center for Specialized Geriatric Care, Nijmegen, the Netherlands
}

Handling Associate Editor: Nagaendran Kandiah

Accepted 2 August 2021

Pre-press 2 September 2021

\begin{abstract}
.
Background: There has been growing interest in young people living with dementia. Future research requires consensus on the terminology and operational definition of this group.

Objective: The purpose of this integrative review was to explore and include all operational definitions used to define dementia at a young age.

Methods: On August 14, 2020, the PubMed, Embase, Cinahl, and PsycInfo databases were searched for empirical and theoretical literature using Google. Various terms to describe and define 'dementia' and 'at a young age' were used to collect literature concerning terminology; age-related aspects, including cut-off ages and criteria; and etiologies of dementia at a young age.

Results: The search yielded 6,891 empirical and 4,660 theoretical publications, resulting in the inclusion of 89 publications, including 36 publications containing an explicit discussion and 53 publications as confirmation. 'Young-onset dementia' was the most commonly used term of seven identified terms, in the last two decades. The age of 65 years at symptom onset was used most frequently when considering a total of six upper age limits and four criteria to define a cut-off age. Eight lower age limits and an option for subdivision based on age were included. We identified 251 different etiologies and 27 categories of etiologies.

Conclusion: Despite relative consensus on the term young-onset dementia and an age at symptom onset being used as a cut-off criterion, much is still unclear concerning possible etiologies of dementia at a young age. In the current study, controversies were detected for discussion in an international consensus study.
\end{abstract}

Keywords: Definition, operationalization, young-onset dementia

\footnotetext{
*Correspondence to: Christian Bakker, PhD, P.O. Box 9101, 6500 HB Nijmegen, the Netherlands. E-mail: Christian.Bakker@ Radboudumc.nl.
} 


\section{INTRODUCTION}

Dementia is often associated with old age but also occurs in younger people. There is little agreement in the scientific literature regarding the terminology and definition of this younger group. The number of young people living with dementia is substantial, as it is estimated that $6 \%-9 \%$ of all people living with dementia worldwide are below the age of 65 [1].

Historically, the first term to describe people living with dementia at a younger age was presenile dementia. This term was introduced by Aloï Alzheimer, who described a 51-year-old patient, Aguste D., showing signs of dementia caused by atypical arteriosclerosis of the brain [2]. In recent decades, other terms have been introduced into the literature, such as early-onset dementia and young-onset dementia. As these terms are used interchangeably in the literature, this can cause ambiguity, as it is unclear to what etiologies and to whom exactly these terms refer. Some authors have suggested the use of different terms to refer to specific subgroups within the group of people living with dementia at a young age $[3,4]$.

In addition to terminology issues, recent research has focused on gaining insight into the characteristics and care needs of young people living with dementia and how they differ from those living with dementia in old age. The distinction between dementia at a young versus an old age is important from a research and care perspective. Considering the first perspective, there is greater heterogeneity in the potential causes of dementia in young versus old individuals and differences in both the course and characteristics of the disease. These causes range from Alzheimer's disease to less-common causes, such as metabolic disorders and prion diseases [5, 6]. Regarding disease course, there is evidence suggesting that differences exist in the disease mechanism of Alzheimer's disease in the brains of young people compared to old people $[7,8]$. Furthermore, characteristics of the disease differ from late onset Alzheimer's disease, as other regions in the brain tend to be involved at a younger age, resulting in phenotypic variants, such as logopenic variant primary progressive aphasia, posterior cortical atrophy, and corticobasal degeneration [9], more often causing nonanamnestic presentations [10]. This might also explain why young individuals with Alzheimer's disease have more intact disease awareness than people with Alzheimer's disease in old age, which appears to be a risk factor for developing depressive symptoms in younger individuals with Alzheimer's disease [11]. In frontotemporal dementia, typically occurring in younger individuals, disinhibition and absent disease awareness are among the key symptoms [12]. From a care perspective, early symptoms of dementia at a young age are often not recognized as such by general practitioners, leading to a delay in a timely referral to appropriate diagnostic services and a consequential delay in the initiation of appropriate services and support [13]. Furthermore, the care needs of young individuals with dementia differ from those of older people living with dementia [14]. For instance, younger people with dementia live active lives, leading to specific needs, such as daytime activities, mobility, and information. They also express the need to retain a sense of usefulness and stay engaged [15] as well as in some cases provide care for elderly parents themselves. Care costs almost doubles in case of dementia onset before retirement [16] and financial issues occur commonly [17]. These differences in care needs suggest that tailored care and specialized care services for younger people with dementia and their caregivers alike are needed [14]. In several countries, this has led to the development of dedicated services for this particular group; however, these services are scarce and difficult to access, especially when-in some countries-care services are restricted for people over a certain age [18].

To distinguish between younger and older individuals living with dementia, the age of 65 is typically used as the cut-off age [6], as this is the retirement age in many countries. However, in the literature, other cut-off ages are also used, such as the age of 60 years, justified by evidence suggesting a role change of $A P O E \varepsilon 4$ in the cause of Alzheimer's disease around this age [19], or 70 years.

In summary, various differences in etiologies, phenotypes and care needs exist, together with both a delay in timely referrals to receive a proper diagnosis and scarce availability of age-specific post-diagnostic support. A clear operational definition is necessary, yet lacking, to conduct fundamental, clinical, and translational research in the field of dementia at a young age. Therefore, the aim of this integrative review was to explore and include all operational definitions used to define dementia at a young age.

\section{METHODS}

This integrative review was part of the larger Prevalence REcognition and Care pathways in young Onset DEmentia (PRECODE) project on the prevalence, incidence, definition, and care pathways of dementia 
at a young age. Integrative reviews concentrate on both empirical scientific literature and theoretical documents. The larger diversity in the sampling frame in an integrative review allows for a broad input of relevant data, which provides a more comprehensive understanding in the case of a complex topic in health care [20]. A five-stage framework developed to guide the process of conducting integrative reviews was applied, focusing on problem identification, the literature search itself, data evaluation, data analysis, and presentation of the results [21].

\section{Literature search}

On August 14, 2020, the PubMed, Embase, Cinahl, and PsycInfo databases were searched for empirical literature. Various terms to describe 'dementia' were included in the search strategy supplemented with various terms to describe 'at a young age' (for search terms, see the Supplementary Material 1). Theoretical literature (i.e., non-empirical literature such as local dementia strategies) was searched using www.google.com, adding a third term to focus on 'guidelines, policy documents and strategy documents', to ensure coverage of all available terms and definitions. The goal was to include literature published by governments, public agencies, professional or provider organizations, industry, and advocacy groups. Regardless of the database, all relevant empirical and theoretical literature was included. Only literature in the English and Dutch languages was included.

\section{Data evaluation}

After removing duplicate publications, all empirical literature was screened using the title and abstract. The literature was selected, and labelled 'category 1 literature', when one or more of the following aspects were explicitly discussed: 1) terminology or nomenclature; 2) age-related aspects, such as cutoff ages and criteria; and 3) etiologies, diseases or disorders that may cause dementia at a young age. Literature that described but did not discuss one or more of these three aspects was labelled 'category 2 literature'. For the selection procedure of empirical literature, Rayyan (http://rayyan.qcri.org) was used. Rayyan is a user-friendly, time-efficient web based tool that enables one or multiple people, if desired double-blinded, to allocate titles or titles and abstracts to custom made categories [22].
Theoretical literature was screened and selected manually. The literature was sorted based on relevance in Google using the 'sort on relevance' button. The full text literature was screened for relevant content, starting with 50 publications. When new aspects, i.e., terms, age-related aspects, or etiologies, emerged in these 50 publications, an additional 50 publications were included until no new relevant aspects were found. Only theoretical literature containing new information relative to empirical literature was included. The reference lists of the included literature were manually reviewed for additional literature.

Initial screening was performed by the first author (DvdV). To safeguard the quality of the selection process, the second author (CB) independently screened the first 200 titles and abstracts of empirical literature, all category 1 literature and the first 20 theoretical publications. Differences were discussed in consensus meetings between DV and CB.

The full text of all 'category 1 literature' was read by the first author (DvdV) to gain insight in the relevance of the data and how it was organized in the article. Then the data was extracted and third, the rationale of the choices made by the authors was captured. The first author also verified the category label assigning of the previous step. Additionally, to establish whether there were terms used in the theoretical literature, above and beyond those found in the empirical literature and to gain insight into the frequency of the terms and definitions, the full text of a random sample of $10 \%$ of all category 2 empirical literature was also read by DvdV.

\section{Data analysis}

A data extraction form was used to summarize the data from the primary literature and quality assessment scores by DvdV (Table 1). To our knowledge, no quality checklist exists to assess both empirical and theoretical literature. Therefore, a four-scale checklist was compiled assessing the authenticity, methodological quality, informational value, and representativity of the primary sources (Supplementary Material 2), an approach endorsed by Whittemore and Knafl [20]. The checklist was based on the Critical Appraisal Skills Programme [23] and the Authority Accuracy Coverage Objectivity Date Significance (AACODS) checklist [24], which are quality checklists for empirical and theoretical literature, respectively. Category 1 literature was appraised and rated as good $(+)$, adequate $( \pm)$, or inadequate $(-)$. 
Table 1

Category 1 terminology and definitions of dementia at a young age

\begin{tabular}{|c|c|c|c|c|c|c|c|c|c|c|}
\hline \multirow[t]{2}{*}{ Authors (y) } & \multicolumn{3}{|c|}{ Study } & \multirow[t]{2}{*}{ Term(s) } & \multicolumn{3}{|c|}{ Age-related aspects } & \multicolumn{2}{|c|}{ Etiologies $\dagger$} & \multirow{2}{*}{$\begin{array}{l}\text { Quality } \\
+, \pm \text {, or }-\end{array}$} \\
\hline & Design & $\begin{array}{l}\text { Population } \\
\text { (n) }\end{array}$ & Setting & & $\begin{array}{l}\text { Upper age } \\
\text { limit }\end{array}$ & $\begin{array}{l}\text { Lower age } \\
\text { limit }\end{array}$ & Subdivision & Diseases $\ddagger$ & Categories & \\
\hline $\begin{array}{l}\text { Ahmed et al. } \\
\text { (2016) [50] }\end{array}$ & $\begin{array}{l}\text { Symposium } \\
\text { paper }\end{array}$ & $\mathrm{n} / \mathrm{a}$ & $\mathrm{n} / \mathrm{a}$ & $\begin{array}{l}\text { Young-onset } \\
\text { dementia }\end{array}$ & 65 & $\mathrm{n} / \mathrm{a}$ & $\mathrm{n} / \mathrm{a}$ & $\begin{array}{l}\text { 1, 1a, 2a, 2b, 2c-i, } \\
\text { 2c-ii, 2c-iii, 3, 4, 5, } \\
6,7,8,29 \mathrm{a}, 30,31, \\
33,35,39,42 \mathrm{a}, 43, \\
43 \mathrm{a}, 43 \mathrm{c}, 45,46, \\
58 \mathrm{a}, 60,62,64,67, \\
69,71,73,77,78 \mathrm{a}, \\
80,82,86,87,111, \\
133,135,136,145, \\
147,148 \mathrm{a}\end{array}$ & $\begin{array}{l}\text { I, II, III, IV, IVa, } \\
\text { Va, Vb, Vc, } \\
\text { IXc, XI }\end{array}$ & Good \\
\hline $\begin{array}{l}\text { Charlton } \\
\text { (1975) [52] }\end{array}$ & Opinion & $\mathrm{n} / \mathrm{a}$ & $\mathrm{n} / \mathrm{a}$ & $\begin{array}{l}\text { Presenile } \\
\text { dementia }\end{array}$ & $\mathrm{n} / \mathrm{a}$ & $\mathrm{n} / \mathrm{a}$ & $\mathrm{n} / \mathrm{a}$ & $\begin{array}{l}1,2,4,5,8,10,14,29, \\
31,33,46,58 \mathrm{a}, 60, \\
73 \mathrm{f}, 73 \mathrm{~g}, 76,77 \mathrm{~b}, \\
77 \mathrm{~h}, 78,78 \mathrm{a}, 78 \mathrm{~b}, \\
78 \mathrm{~d}, 80,87,133, \\
135,136,145,149, \\
150,152,153,157, \\
160\end{array}$ & $\begin{array}{l}\text { II, IV, Va, Vb, } \\
\text { IXb, IXc, XIV }\end{array}$ & Good \\
\hline $\begin{array}{l}\text { Coblentz et al. } \\
\text { (1973) [65] }\end{array}$ & $\begin{array}{l}\text { Prospective } \\
\text { study }\end{array}$ & 30 & Hospital & $\begin{array}{l}\text { Presenile } \\
\text { dementia }\end{array}$ & 65 & $\mathrm{n} / \mathrm{a}$ & $\mathrm{n} / \mathrm{a}$ & $1,2,32,40,58 \mathrm{a}, 135$ & IV, X & Good \\
\hline $\begin{array}{l}\text { Cowie (1981) } \\
{[66]}\end{array}$ & Opinion & $\mathrm{n} / \mathrm{a}$ & $\mathrm{n} / \mathrm{a}$ & $\begin{array}{l}\text { Presenile } \\
\text { dementia }\end{array}$ & $\mathrm{n} / \mathrm{a}$ & $\mathrm{n} / \mathrm{a}$ & $\mathrm{n} / \mathrm{a}$ & $1,2,8,58 \mathrm{a}, 87$ & $\mathrm{n} / \mathrm{a}$ & Good \\
\hline $\begin{array}{l}\text { Davies et al. } \\
\text { (2011) [25] }\end{array}$ & Review & $\mathrm{n} / \mathrm{a}$ & $\mathrm{n} / \mathrm{a}$ & $\begin{array}{l}\text { Presenile } \\
\text { dementia, } \\
\text { early-onset } \\
\text { dementia }\end{array}$ & $\begin{array}{l}65 \text { at symptom } \\
\text { onset }\end{array}$ & $\mathrm{n} / \mathrm{a}$ & $\mathrm{n} / \mathrm{a}$ & $\begin{array}{l}\text { 1, 2, 2a, 2c-i, 2c-ii, 30, } \\
31,33,34,39 \\
\text { 42a-ii, 43b, 57, 58, } \\
\text { 58a, 61a, 73, 73c, } \\
77 \mathrm{e}, 86,133,135, \\
136,138,147,150 \\
151,152,159\end{array}$ & $\begin{array}{l}\text { I, II, III, IV, Vb, } \\
\text { IX, X, XI, } \\
\text { XIIb }\end{array}$ & Good \\
\hline $\begin{array}{l}\text { Dixon } \\
\text { (2007) [37] }\end{array}$ & Opinion & $\mathrm{n} / \mathrm{a}$ & $\mathrm{n} / \mathrm{a}$ & $\begin{array}{l}\text { Early-onset } \\
\text { dementia, } \\
\text { young-onset } \\
\text { dementia }\end{array}$ & 60,65 & $\mathrm{n} / \mathrm{a}$ & $\mathrm{n} / \mathrm{a}$ & 1 & $\mathrm{n} / \mathrm{a}$ & Adequate \\
\hline
\end{tabular}




\begin{tabular}{|c|c|c|c|c|c|c|c|c|c|c|}
\hline $\begin{array}{l}\text { Doran } \\
\text { (1997) [41] }\end{array}$ & Opinion & $\mathrm{n} / \mathrm{a}$ & $\mathrm{n} / \mathrm{a}$ & $\begin{array}{l}\text { Presenile } \\
\text { dementia }\end{array}$ & 65 at diagnosis & 16 & $\mathrm{n} / \mathrm{a}$ & $\begin{array}{l}1,2,2 \mathrm{~d}, 3,4,5,6,7,8, \\
9,10,18,19,20,26, \\
28,29,29 \mathrm{a}, 29 \mathrm{~b}, 30, \\
31,35,35 \mathrm{c}, 37,39, \\
41,42 \mathrm{a}, 43,43 \mathrm{a}, \\
43 \mathrm{~b}, 43 \mathrm{e}, 43 \mathrm{f}, 43 \mathrm{~g}, \\
45,51,54,57,58 \mathrm{a}, \\
58 \mathrm{c}, 59,60,62,64, \\
67,69,72,73,73 \mathrm{c}, \\
73 \mathrm{e}, 75,76,77 \mathrm{~g}, 78, \\
87,88,91,93,103, \\
110,112,113,114, \\
115,116,117,118, \\
120,124,125,126, \\
130,131,132,133, \\
135,140,141,142, \\
146\end{array}$ & $\begin{array}{l}\text { I, II, III, IV, Va, } \\
\text { Vb, }\end{array}$ & Good \\
\hline $\begin{array}{l}\text { Draper and } \\
\text { Withall } \\
\text { (2016) [32] }\end{array}$ & Review & $\mathrm{n} / \mathrm{a}$ & $\mathrm{n} / \mathrm{a}$ & $\begin{array}{l}\text { Young onset } \\
\text { dementia }\end{array}$ & $\begin{array}{l}65 \text { at symptom } \\
\text { onset }\end{array}$ & $\mathrm{n} / \mathrm{a}$ & $\begin{array}{l}30-44, \\
45-64\end{array}$ & $\begin{array}{l}1,2,3,4,5,6,8,12, \\
14,14 \mathrm{a}, 29,30,39, \\
42 \mathrm{a}, 43 \mathrm{~b}, 43 \mathrm{c}, 57, \\
58 \mathrm{a}, 60,67,73,73 \mathrm{c}, \\
87,109,110,111, \\
112,135,162 \ddagger\end{array}$ & $\begin{array}{l}\text { I, IV, Vb, IXc, } \\
\text { XV }\end{array}$ & Good \\
\hline $\begin{array}{l}\text { Fadil et al. } \\
\text { (2009) [49] }\end{array}$ & Review & $\mathrm{n} / \mathrm{a}$ & $\mathrm{n} / \mathrm{a}$ & $\begin{array}{l}\text { Early-onset } \\
\text { dementia }\end{array}$ & $\begin{array}{l}65 \text { at symptom } \\
\text { onset }\end{array}$ & $\mathrm{n} / \mathrm{a}$ & $\mathrm{n} / \mathrm{a}$ & $\begin{array}{l}\text { 1, 2, 2a, 2b, 2c-i, 2c-ii, } \\
3,4,5,6,7,8,10 \\
12,14,16,19,23, \\
29,29 \mathrm{a}, 29 \mathrm{~b}, 30,31, \\
33,33 \mathrm{a}, 39,41,42 \\
42 \mathrm{a}, 43,43 \mathrm{a}, 43 \mathrm{~b}, \\
43 \mathrm{c}, 45,46,49,50, \\
57,58,58 \mathrm{a}, 58 \mathrm{~b}, \\
\text { 58c, 58d, 59, 60, 61, } \\
62,67,69,70,71, \\
72,73 \mathrm{c}, 73 \mathrm{e}, 73 \mathrm{f}, 76, \\
77,77 \mathrm{~b}, 77 \mathrm{c}, 77 \mathrm{f}, \\
78 \mathrm{a}, 78 \mathrm{c}, 82,84,86, \\
87,88,89,90,133 \\
134,135,136,139 \\
140,141,142,143, \\
145,148,151,152 \\
153,155,161,167\end{array}$ & $\begin{array}{l}\text { I, II, IV, Va-Vb, } \\
\text { VI, VIII, IXc, } \\
\text { X, XI, XV }\end{array}$ & Good \\
\hline
\end{tabular}


Table 1

(Continued)

\begin{tabular}{|c|c|c|c|c|c|c|c|c|c|c|}
\hline \multirow[t]{2}{*}{ Authors (y) } & \multicolumn{3}{|c|}{ Study } & \multirow[t]{2}{*}{ Term(s) } & \multicolumn{3}{|c|}{ Age-related aspects } & \multicolumn{2}{|c|}{ Etiologies $\dagger$} & \multirow{2}{*}{$\begin{array}{r}\text { Quality } \\
+, \pm \text {, or }-\end{array}$} \\
\hline & Design & $\begin{array}{l}\text { Population } \\
\text { (n) }\end{array}$ & Setting & & $\begin{array}{l}\text { Upper age } \\
\text { limit }\end{array}$ & $\begin{array}{l}\text { Lower age } \\
\text { limit }\end{array}$ & Subdivision & Diseases $\ddagger$ & Categories & \\
\hline $\begin{array}{l}\text { Forbes } \\
(2005) \text { [67] }\end{array}$ & Book review & $\mathrm{n} / \mathrm{a}$ & $\mathrm{n} / \mathrm{a}$ & $\begin{array}{l}\text { Presenile } \\
\text { dementia, } \\
\text { early-onset } \\
\text { dementia }\end{array}$ & 65 & $\mathrm{n} / \mathrm{a}$ & $\mathrm{n} / \mathrm{a}$ & $\begin{array}{l}1,2,2 \mathrm{c}-\mathrm{i}, 2 \mathrm{c}-\mathrm{ii}, 3,8 \\
58,58 \mathrm{a}\end{array}$ & III & Good \\
\hline $\begin{array}{l}\text { Giannakopoulos } \\
\text { et al. (1996) } \\
\text { [68] }\end{array}$ & Review & $\mathrm{n} / \mathrm{a}$ & $\mathrm{n} / \mathrm{a}$ & $\begin{array}{l}\text { Early-onset } \\
\text { dementia }\end{array}$ & $\begin{array}{l}65 \text { at } \\
\text { symptom } \\
\text { onset }\end{array}$ & $\mathrm{n} / \mathrm{a}$ & $\mathrm{n} / \mathrm{a}$ & $1,2,2 \mathrm{e}$ & $\begin{array}{l}\text { I, II, III, IXb, } \\
\text { IXc }\end{array}$ & Good \\
\hline $\begin{array}{l}\text { Gibson } \\
(1960) \text { [42] }\end{array}$ & Opinion & $\mathrm{n} / \mathrm{a}$ & $\mathrm{n} / \mathrm{a}$ & $\begin{array}{l}\text { Presenile } \\
\text { dementia }\end{array}$ & 65 & $\begin{array}{l}\text { Covers from } \\
40, \text { occurs } \\
\text { in much } \\
\text { younger }\end{array}$ & $\mathrm{n} / \mathrm{a}$ & $1,2,8,58 \mathrm{a}$ & $\mathrm{n} / \mathrm{a}$ & Adequate \\
\hline $\begin{array}{l}\text { Goldman } \\
\text { (2015) [38] }\end{array}$ & Review & $\mathrm{n} / \mathrm{a}$ & $\mathrm{n} / \mathrm{a}$ & $\begin{array}{l}\text { Early-onset } \\
\text { dementia, } \\
\text { young-onset } \\
\text { dementia }\end{array}$ & 60 & $\mathrm{n} / \mathrm{a}$ & $\mathrm{n} / \mathrm{a}$ & $\begin{array}{l}1,2,2 \mathrm{a}, 3,4,8,9,12, \\
14,19,29,30,58, \\
58 \mathrm{a}, 58 \mathrm{~b}, 58 \mathrm{c}, 59 \\
87,104,108,110 \\
111,112,120,121, \\
125,126,130\end{array}$ & $\mathrm{I}, \mathrm{Vb}, \mathrm{VI}, \mathrm{XVI}$ & Good \\
\hline $\begin{array}{l}\text { Goodall } \\
\text { (1938) [43] }\end{array}$ & Discussion & $\mathrm{n} / \mathrm{a}$ & $\mathrm{n} / \mathrm{a}$ & $\begin{array}{l}\text { Presenile } \\
\text { dementia }\end{array}$ & $\begin{array}{l}60 \text { at } \\
\text { symptom } \\
\text { onset }\end{array}$ & Usually 40 & $\mathrm{n} / \mathrm{a}$ & $\begin{array}{l}1,2,8,39,58 \mathrm{a}, 60 \\
60 \mathrm{a}, 60 \mathrm{~b}, 66,73 \\
73 \mathrm{~d}, 73 \mathrm{e}, 77 \mathrm{a}, 77 \mathrm{~b}, \\
78 \mathrm{a}, 79,80,133 \\
136,137,147\end{array}$ & $\begin{array}{l}\text { II, Va, IXb, XIV, } \\
\text { XVII, XVIII, } \\
\text { XIX }\end{array}$ & Adequate \\
\hline $\begin{array}{l}\text { Greicius et al. } \\
\text { (2002) [26] }\end{array}$ & Review & $\mathrm{n} / \mathrm{a}$ & $\mathrm{n} / \mathrm{a}$ & $\begin{array}{l}\text { Presenile } \\
\text { dementia }\end{array}$ & 60 & $\mathrm{n} / \mathrm{a}$ & $\mathrm{n} / \mathrm{a}$ & $\begin{array}{l}1,2,2 \mathrm{a}, 2 \mathrm{c}-\mathrm{i}, 2 \mathrm{c}-\mathrm{ii}, 3 \\
5,6,58,58 \mathrm{a}, 58 \mathrm{c}, \\
58 \mathrm{~d}, 59\end{array}$ & $\mathrm{n} / \mathrm{a}$ & Good \\
\hline $\begin{array}{l}\text { Harvey } \\
(1998) \text { [29] }\end{array}$ & $\begin{array}{l}\text { Theoretical } \\
\text { thesis }\end{array}$ & $\mathrm{n} / \mathrm{a}$ & $\mathrm{n} / \mathrm{a}$ & $\begin{array}{l}\text { Young onset } \\
\text { dementia, } \\
\text { presenile } \\
\text { dementia }\end{array}$ & $\begin{array}{l}60,65,70 \text {, at } \\
\text { symptom } \\
\text { onset, } \\
\text { diagnosis, at } \\
\text { presentation } \\
\text { to medical } \\
\text { services }\end{array}$ & 30,35 & $\begin{array}{l}30-44 \\
45-64\end{array}$ & $\begin{array}{l}1,2,3,4,5,6,8,29 \\
29 \mathrm{a}, 30,31,33,39 \\
57,58,58 \mathrm{a}, 58 \mathrm{~b}, 73 \\
73 \mathrm{c}, 73 \mathrm{e}, 73 \mathrm{f}, 73 \mathrm{~g}, \\
82,135,161,169 \\
172\end{array}$ & $\mathrm{n} / \mathrm{a}$ & Good \\
\hline
\end{tabular}




\begin{tabular}{|c|c|c|c|c|c|c|c|c|c|c|}
\hline $\begin{array}{l}\text { Hayo } \\
\text { (2015) [34] }\end{array}$ & Review & $\mathrm{n} / \mathrm{a}$ & $\mathrm{n} / \mathrm{a}$ & $\begin{array}{l}\text { Young-onset } \\
\text { dementia, } \\
\text { younger } \\
\text { people with } \\
\text { dementia, } \\
\text { early-onset } \\
\text { dementia, } \\
\text { working age } \\
\text { dementia }\end{array}$ & $\begin{array}{l}65 \text { year at } \\
\text { diagnosis }\end{array}$ & $\mathrm{n} / \mathrm{a}$ & $\begin{array}{l}35-45 \\
45-65\end{array}$ & $\begin{array}{l}1,1 \mathrm{a}, 2,3,4,5,6,8,29 \\
39,57,58 \mathrm{a}, 73,110 \\
135,150,131,161\end{array}$ & $\begin{array}{l}\text { I, IVa, } \mathrm{Vb}, \mathrm{Vc}, \\
\mathrm{XX}\end{array}$ & $\overline{\text { Good }}$ \\
\hline $\begin{array}{l}\text { Koopmans and } \\
\text { Rosness } \\
\text { (2014) [35] }\end{array}$ & Editorial & $\mathrm{n} / \mathrm{a}$ & $\mathrm{n} / \mathrm{a}$ & $\begin{array}{l}\text { Presenile } \\
\text { dementia, } \\
\text { early-onset } \\
\text { dementia, } \\
\text { younger-onset } \\
\text { dementia, } \\
\text { young-onset } \\
\text { dementia }\end{array}$ & $\begin{array}{l}65,45 \text { at } \\
\text { symptom } \\
\text { onset or at } \\
\text { diagnosis }\end{array}$ & 17 & $\mathrm{n} / \mathrm{a}$ & 1 & $\mathrm{n} / \mathrm{a}$ & Good \\
\hline $\begin{array}{l}\text { Kuruppu and } \\
\text { Matthews } \\
\text { (2013) [4] }\end{array}$ & Review & $\mathrm{n} / \mathrm{a}$ & $\mathrm{n} / \mathrm{a}$ & $\begin{array}{l}\text { Young-onset } \\
\text { dementia }\end{array}$ & 65 & Typically 45 & $\mathrm{n} / \mathrm{a}$ & $\begin{array}{l}1,2,3,7,8,17,29,30, \\
35,39,42,42 \mathrm{a}, 43 \mathrm{a}, \\
45,57,58 \mathrm{a}, 60,67, \\
71,73,73 \mathrm{e}, 73 \mathrm{f}, 77, \\
78,81,86,87,104, \\
105,106,108,109 \mathrm{a}, \\
109 \mathrm{~b}, 110,111,112, \\
120,121,122,123, \\
124,125,126,145, \\
147,150,151,152, \\
157 \ddagger\end{array}$ & $\begin{array}{l}\text { III, IV, Va, Vc, } \\
\text { VI, VII, VIII, } \\
\text { XI }\end{array}$ & Good \\
\hline $\begin{array}{l}\text { Laino } \\
\text { (2005) [69] }\end{array}$ & $\begin{array}{l}\text { Interview } \\
\text { about } \\
\text { prospec- } \\
\text { tive } \\
\text { study }\end{array}$ & 278 & Home & $\begin{array}{l}\text { Early-onset } \\
\text { dementia }\end{array}$ & $\begin{array}{l}65 \text { at symptom } \\
\text { onset }\end{array}$ & $\mathrm{n} / \mathrm{a}$ & $\mathrm{n} / \mathrm{a}$ & $\begin{array}{l}1,2,57,60,73,73 f \\
134,135,151\end{array}$ & $\mathrm{n} / \mathrm{a}$ & Adequate \\
\hline $\begin{array}{l}\text { Levine } \\
\text { (2013) [70] }\end{array}$ & Opinion & $\mathrm{n} / \mathrm{a}$ & $\mathrm{n} / \mathrm{a}$ & $\begin{array}{l}\text { Young-onset } \\
\text { dementia }\end{array}$ & 65 & $\mathrm{n} / \mathrm{a}$ & $\mathrm{n} / \mathrm{a}$ & $\mathrm{n} / \mathrm{a}$ & $\begin{array}{l}\text { I, II, III, IV, Vb, } \\
\text { XIII, XV }\end{array}$ & Good \\
\hline $\begin{array}{l}\text { Liebson et al. } \\
\text { (2005) [51] }\end{array}$ & Opinion & $\mathrm{n} / \mathrm{a}$ & $\mathrm{n} / \mathrm{a}$ & $\begin{array}{l}\text { Early-onset } \\
\text { dementia }\end{array}$ & $\mathrm{n} / \mathrm{a}$ & $\mathrm{n} / \mathrm{a}$ & $\mathrm{n} / \mathrm{a}$ & $\begin{array}{l}1,2,3,4,8,9,29,39, \\
46,57,58 \mathrm{a}, 60,73, \\
73 \mathrm{f}, 77,134,135, \\
147,147 \mathrm{a}, 153,154, \\
157,159,165,166, \\
168\end{array}$ & XVIII, XXI & Good \\
\hline
\end{tabular}


Table 1

\begin{tabular}{|c|c|c|c|c|c|c|c|c|c|c|}
\hline \multirow[t]{2}{*}{ Authors (y) } & \multicolumn{3}{|c|}{ Study } & \multirow[t]{2}{*}{ Term(s) } & \multicolumn{3}{|c|}{ Age-related aspects } & \multicolumn{2}{|c|}{ Etiologies $\dagger$} & \multirow{2}{*}{$\begin{array}{l}\text { Quality } \\
+, \pm \text {, or }-\end{array}$} \\
\hline & Design & $\begin{array}{l}\text { Population } \\
\text { (n) }\end{array}$ & Setting & & $\begin{array}{l}\text { Upper age } \\
\text { limit }\end{array}$ & $\begin{array}{l}\text { Lower age } \\
\text { limit }\end{array}$ & Subdivision & Diseases $\ddagger$ & Categories & \\
\hline $\begin{array}{l}\text { Masellis } \\
\text { et al. (2013) } \\
\text { [28] }\end{array}$ & Review & $\mathrm{n} / \mathrm{a}$ & $\mathrm{n} / \mathrm{a}$ & $\begin{array}{l}\text { Early-onset } \\
\text { dementia, } \\
\text { young-onset } \\
\text { dementia }\end{array}$ & $\begin{array}{l}45,65 \text { year at } \\
\text { symptom } \\
\text { onset }\end{array}$ & $\begin{array}{l}\text { Early adult- } \\
\text { hood }\end{array}$ & $\begin{array}{l}30-45 \\
45-65\end{array}$ & $\begin{array}{l}1,2,2 \mathrm{a}, 2 \mathrm{c}, 2 \mathrm{c}-\mathrm{i}, 2 \mathrm{c}-\mathrm{ii}, \\
2 \mathrm{c}-\mathrm{iii}, 3,4,5,6,10, \\
14,14 \mathrm{a}, 19,29 \mathrm{a}, 30, \\
34,35,42,42 \mathrm{a}, 43, \\
43 \mathrm{~b}, 44,49,57,60, \\
62,73 \mathrm{c}, 73 \mathrm{f}, 86,87, \\
104,108,109,110, \\
111,112,114,120, \\
121,126,129,130, \\
133,140,147 \mathrm{~b}, \\
153 \ddagger\end{array}$ & $\begin{array}{l}\text { I, II, III, IV, Vb, } \\
\text { Vc, IXc, X, XI }\end{array}$ & Good \\
\hline $\begin{array}{l}\text { Miyoshi } \\
\text { (2009) [3] }\end{array}$ & Review & $\mathrm{n} / \mathrm{a}$ & $\mathrm{n} / \mathrm{a}$ & $\begin{array}{l}\text { Presenile } \\
\text { dementia, } \\
\text { early-onset } \\
\text { dementia }\end{array}$ & $\begin{array}{l}65 \text { at symptom } \\
\text { onset }\end{array}$ & $\mathrm{n} / \mathrm{a}$ & $\mathrm{n} / \mathrm{a}$ & $\begin{array}{l}1,2,2 \mathrm{c}-\mathrm{i}, 2 \mathrm{c}-\mathrm{ii}, 5,6 \\
17,29,30,35 \mathrm{a}, 35 \mathrm{~g} \\
57,70,134\end{array}$ & $\mathrm{I}, \mathrm{II}, \mathrm{Va}, \mathrm{Vb}, \mathrm{IX}$ & Good \\
\hline $\begin{array}{l}\text { Moffie } \\
(1953) \text { [44] }\end{array}$ & Review & $\mathrm{n} / \mathrm{a}$ & $\mathrm{n} / \mathrm{a}$ & $\begin{array}{l}\text { Presenile } \\
\text { dementia }\end{array}$ & 65 & 45 & $\mathrm{n} / \mathrm{a}$ & $1,2,8,48,168 \mathrm{a}$ & II & Adequate \\
\hline $\begin{array}{l}\text { NHMRC } \\
\text { National } \\
\text { Institute for } \\
\text { Dementia } \\
\text { Research } \\
\text { (2020) [33] }\end{array}$ & $\begin{array}{l}\text { Theoretical } \\
\text { abstract } \\
\text { book }\end{array}$ & $\mathrm{n} / \mathrm{a}$ & $\mathrm{n} / \mathrm{a}$ & $\begin{array}{l}\text { Young-onset } \\
\text { dementia, } \\
\text { younger-onset } \\
\text { dementia, } \\
\text { early-onset } \\
\text { dementia, } \\
\text { adult-onset } \\
\text { dementia, } \\
\text { childhood } \\
\text { dementia }\end{array}$ & $\begin{array}{l}65, \text { at } \\
\text { symptom } \\
\text { onset, } \\
\text { before } \\
\text { deceased } \\
\text { ("died aged } \\
\text { less than") }\end{array}$ & $\mathrm{n} / \mathrm{a}$ & $\mathrm{n} / \mathrm{a}$ & $1,2,2 \mathrm{a}, 3,4,110,161$ & I & Good \\
\hline $\begin{array}{l}\text { Panegyres } \\
\text { et al. (2000) } \\
\text { [71] }\end{array}$ & $\begin{array}{l}\text { Letter to the } \\
\text { editor }\end{array}$ & $\mathrm{n} / \mathrm{a}$ & $\mathrm{n} / \mathrm{a}$ & $\begin{array}{l}\text { Early-onset } \\
\text { dementia }\end{array}$ & $\begin{array}{l}65 \text { at symptom } \\
\text { onset }\end{array}$ & $\mathrm{n} / \mathrm{a}$ & $\mathrm{n} / \mathrm{a}$ & $\begin{array}{l}1,2,2 \mathrm{a}, 2 \mathrm{c}, 2 \mathrm{c}-\mathrm{i}, 3,4 \\
5,29,57,58 \mathrm{a}, 59 \\
73,159\end{array}$ & XIIb & Good \\
\hline $\begin{array}{l}\text { Pinhorn } \\
\text { (1993) [45] }\end{array}$ & $\begin{array}{l}\text { Letters to } \\
\text { the editor }\end{array}$ & $\mathrm{n} / \mathrm{a}$ & $\mathrm{n} / \mathrm{a}$ & $\begin{array}{l}\text { Presenile } \\
\text { dementia }\end{array}$ & 65 & 40 & $\mathrm{n} / \mathrm{a}$ & $1,29,31,73,134$ & $\mathrm{n} / \mathrm{a}$ & Adequate \\
\hline $\begin{array}{l}\text { Poser } \\
(1975) \text { [72] }\end{array}$ & Opinion & $\mathrm{n} / \mathrm{a}$ & $\mathrm{n} / \mathrm{a}$ & $\begin{array}{l}\text { Presenile } \\
\text { dementia }\end{array}$ & $\mathrm{n} / \mathrm{a}$ & $\mathrm{n} / \mathrm{a}$ & $\mathrm{n} / \mathrm{a}$ & $\begin{array}{l}1,2,4,8,29,47,58 \mathrm{a} \\
60,73,73 \mathrm{c}, 73 \mathrm{f}, 77 \mathrm{~b} \\
77 \mathrm{i}, 77 \mathrm{j}, 84,135 \\
150,153,154,169\end{array}$ & $\mathrm{n} / \mathrm{a}$ & Good \\
\hline
\end{tabular}




\begin{tabular}{|c|c|c|c|c|c|c|c|c|c|c|}
\hline $\begin{array}{l}\text { Ridha and } \\
\text { Josephs } \\
\text { (2006) [47] }\end{array}$ & Review & $\mathrm{n} / \mathrm{a}$ & $\mathrm{n} / \mathrm{a}$ & $\begin{array}{l}\text { Young-onset } \\
\text { dementia }\end{array}$ & $\begin{array}{l}65 \text { at symptom } \\
\text { onset }\end{array}$ & $\mathrm{n} / \mathrm{a}$ & $\mathrm{n} / \mathrm{a}$ & $\begin{array}{l}\text { 1, 1a, 2, 2b, 2c, 2c-i, } \\
2 \mathrm{~d}, 2 \mathrm{f}, 3,4,5,6,8, \\
\text { 10, 11, 12,13b, 14, } \\
\text { 15, 16, 17, 18, 19, } \\
20,21,22,23,28, \\
\text { 29, 30, 35a, 35b, } \\
35 \mathrm{c}, 35 \mathrm{~d}, 35 \mathrm{e}, 35 \mathrm{f}, \\
37,38,39,42,42 \mathrm{a}, \\
43,43 \mathrm{a}, 43 \mathrm{~b}, 43 \mathrm{~d}, \\
43 \mathrm{~h}, 41,52,53,54, \\
56,58,60,61 \mathrm{a}, 61 \mathrm{~b}, \\
62,63,64,65,67, \\
68,69,71,72,73, \\
73 \mathrm{e}, 73 \mathrm{f}, 74,75,76, \\
77,77 \mathrm{c}, 77 \mathrm{~h}, 77 \mathrm{k}, \\
771,77 \mathrm{~m}, 78 \mathrm{a}, 78 \mathrm{e}, \\
80,82,83,85,86, \\
87,88,91,92,93, \\
94,96,102,109, \\
110,112,114,117, \\
119,121,123,125, \\
126,127,128,129, \\
130,131,123,136, \\
141,147 \mathrm{a}, 150, \\
152 \mathrm{a}, 152 \mathrm{~b}, 156, \\
157,158,159,162, \\
163,164,170,171\end{array}$ & $\begin{array}{l}\text { IV, Vb, VI, VII, } \\
\text { XIV }\end{array}$ & Good \\
\hline $\begin{array}{l}\text { Risien Russel } \\
\text { (1933) [39] }\end{array}$ & Discussion & $\mathrm{n} / \mathrm{a}$ & $\mathrm{n} / \mathrm{a}$ & $\begin{array}{l}\text { Presenile } \\
\text { dementia }\end{array}$ & $\begin{array}{l}60,65 \text { at } \\
\text { symptom } \\
\text { onset }\end{array}$ & $\begin{array}{l}\text { Usually } 30, \\
40\end{array}$ & $\mathrm{n} / \mathrm{a}$ & $\begin{array}{l}1,2,8,39,66,73,73 \mathrm{a} \\
73 \mathrm{~d}, 80\end{array}$ & $\begin{array}{l}\text { I, II, IV-Va, IXb, } \\
\text { IXc, XVII }\end{array}$ & Adequate \\
\hline $\begin{array}{l}\text { Rossor et al. } \\
\text { (2010) [6] }\end{array}$ & Review & $\mathrm{n} / \mathrm{a}$ & $\mathrm{n} / \mathrm{a}$ & $\begin{array}{l}\text { Young-onset } \\
\text { dementia }\end{array}$ & $\begin{array}{l}65,45 \text { at } \\
\text { symptom } \\
\text { onset }\end{array}$ & 18,30 & $\begin{array}{l}<35,35-45 \\
45-65\end{array}$ & $\begin{array}{l}1,1 \mathrm{a}, 2,2 \mathrm{a}, 2 \mathrm{~b}, 2 \mathrm{c}-\mathrm{i}, \\
\text { 2c-ii, 2d, 2f, 3, 4, 5, } \\
7,8,10,12,13 \mathrm{a}, \\
\text { 13b, 13c, 14, 14a, } \\
\text { 14b, 14c, 14d, 15, } \\
\text { 19, 20, 22, 23, 24, } \\
25,27,30,35,35 \mathrm{a}, \\
35 \mathrm{~b}, 39,42,42 \mathrm{a}, \\
42 \mathrm{~b}, 43 \mathrm{c}, 43 \mathrm{~d}, 49, \\
51,57,58,58 \mathrm{~b}, 59, \\
60,61 \mathrm{a}, 64,69,73, \\
73 \mathrm{f}, 86,87,88,92, \\
94,99,100,101, \\
102,107,109,110, \\
111,112,114,115, \\
117,120,121,122, \\
123,125,126,130, \\
131,145,147 \ddagger\end{array}$ & $\begin{array}{l}\text { III, IV, Va, Vb, } \\
\text { VI, XIII }\end{array}$ & Good \\
\hline
\end{tabular}


Table 1

Continued)

\begin{tabular}{|c|c|c|c|c|c|c|c|c|c|c|}
\hline \multirow[t]{2}{*}{ Authors (y) } & \multicolumn{3}{|c|}{ Study } & \multirow[t]{2}{*}{ Term(s) } & \multicolumn{3}{|c|}{ Age-related aspects } & \multicolumn{2}{|c|}{ Etiologies $\dagger$} & \multirow{2}{*}{$\begin{array}{l}\text { Quality } \\
+, \pm \text {, or - }\end{array}$} \\
\hline & Design & $\begin{array}{l}\text { Population } \\
\text { (n) }\end{array}$ & Setting & & $\begin{array}{l}\text { Upper age } \\
\text { limit }\end{array}$ & $\begin{array}{l}\text { Lower age } \\
\text { limit }\end{array}$ & Subdivision & Diseases $\ddagger$ & Categories & \\
\hline $\begin{array}{l}\text { Sampson et al. } \\
\text { (2004) [5] }\end{array}$ & Review & $\mathrm{n} / \mathrm{a}$ & $\mathrm{n} / \mathrm{a}$ & $\begin{array}{l}\text { Young onset } \\
\text { dementia }\end{array}$ & 60,65 & $20,30,45$ & $45-65$ & $\begin{array}{l}\text { 1, 2, 2a, 2b, 2c-i, 2c-ii, } \\
3,4,5,6,7,8,10, \\
14,15,16,19,20, \\
23,29,29 \mathrm{a}, 30,31, \\
33,35,35 \mathrm{a}, 35 \mathrm{~b}, \\
\text { 35c, 35d, 35e, 39, } \\
41,42 \mathrm{a}, 43,43 \mathrm{a}, \\
43 \mathrm{~b}, 43 \mathrm{c}, 45,46,49, \\
\text { 57, 58a, 58b, 58c, } \\
\text { 59, 60, 61a, 61b, 62, } \\
64,65,67,69,70, \\
71,73,73 \mathrm{c}, 77,77 \mathrm{c}, \\
77 \mathrm{~d}, 78 \mathrm{a}, 80,82,86, \\
87,94,102,109 \mathrm{~b}, \\
110,111,112,114, \\
117,120,121,124, \\
125,126,130,131, \\
133,135,136,141, \\
145,147,161 \ddagger\end{array}$ & $\begin{array}{l}\text { I, II, III, IV, Va, } \\
\text { Vb, Vc, VI, } \\
\text { VII, IXc, XI, } \\
\text { XII }\end{array}$ & Good \\
\hline $\begin{array}{l}\text { Sitek et al. } \\
\text { (2015) [53] }\end{array}$ & Review & $\mathrm{n} / \mathrm{a}$ & $\mathrm{n} / \mathrm{a}$ & $\begin{array}{l}\text { Early-onset } \\
\text { dementia, } \\
\text { young-onset } \\
\text { dementia }\end{array}$ & 65 & $\mathrm{n} / \mathrm{a}$ & $\mathrm{n} / \mathrm{a}$ & $\begin{array}{l}\text { 1, 1a, 2, 2a, 2c, 2c-i, } \\
\text { 2c-ii, 2c-iii, 3, 5, 6, } \\
8,29,135\end{array}$ & $\begin{array}{l}\text { I, IV, Va, VI, X, } \\
\text { XV }\end{array}$ & Good \\
\hline $\begin{array}{l}\text { Tonkonogy and } \\
\text { Moak (1988) } \\
{[36]}\end{array}$ & Opinion & $\mathrm{n} / \mathrm{a}$ & $\mathrm{n} / \mathrm{a}$ & $\begin{array}{l}\text { Presenile } \\
\text { dementia }\end{array}$ & $\begin{array}{l}50,55 \text { at } \\
\text { symptom } \\
\text { onset }\end{array}$ & $\mathrm{n} / \mathrm{a}$ & $\mathrm{n} / \mathrm{a}$ & $1,29,60,60 a, 73 b$ & $\mathrm{n} / \mathrm{a}$ & Good \\
\hline $\begin{array}{l}\text { Werner et al. } \\
\text { (2009) [17] }\end{array}$ & Review & $\mathrm{n} / \mathrm{a}$ & $\mathrm{n} / \mathrm{a}$ & $\begin{array}{l}\text { Presenile } \\
\text { dementia, } \\
\text { early-onset } \\
\text { dementia }\end{array}$ & 65 & $\mathrm{n} / \mathrm{a}$ & $45-65$ & $\begin{array}{l}1,2,3,4,5,8,9,29 \\
43,57,73,77,133 \\
134\end{array}$ & $\mathrm{n} / \mathrm{a}$ & Good \\
\hline
\end{tabular}

$\dagger$ See Table $4 .{ }^{\ddagger}$ Including dementia plus syndromes. 


\section{RESULTS}

\section{Search results}

A total of 6,891 empirical publications were identified, of which 4,090 remained after duplicates were removed (Fig. 1). Screening of the title and abstract reduced the number to 51 , all of which were read in full and resulted in the inclusion of 34 category 1 studies.

A total of 4,660 theoretical publications were found, and after reading the first 50 full texts, two

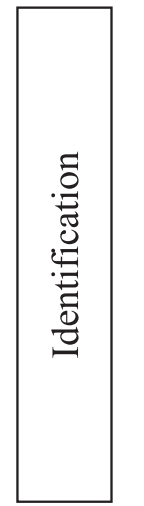

\section{Records identified through database searching} (6,891):

PubMed: $\mathrm{n}=2,278$

Embase: $n=2,143$

Cinahl: $\mathrm{n}=910$

PsycInfo: $\mathrm{n}=1,560$
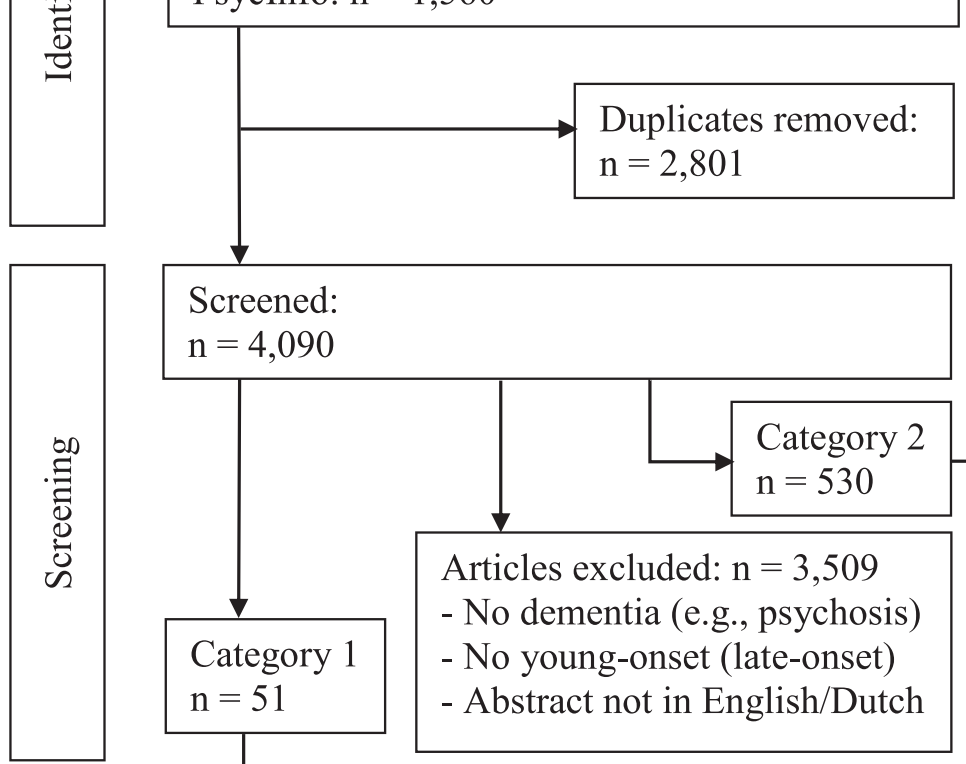

Screened:

$\mathrm{n}=4,090$
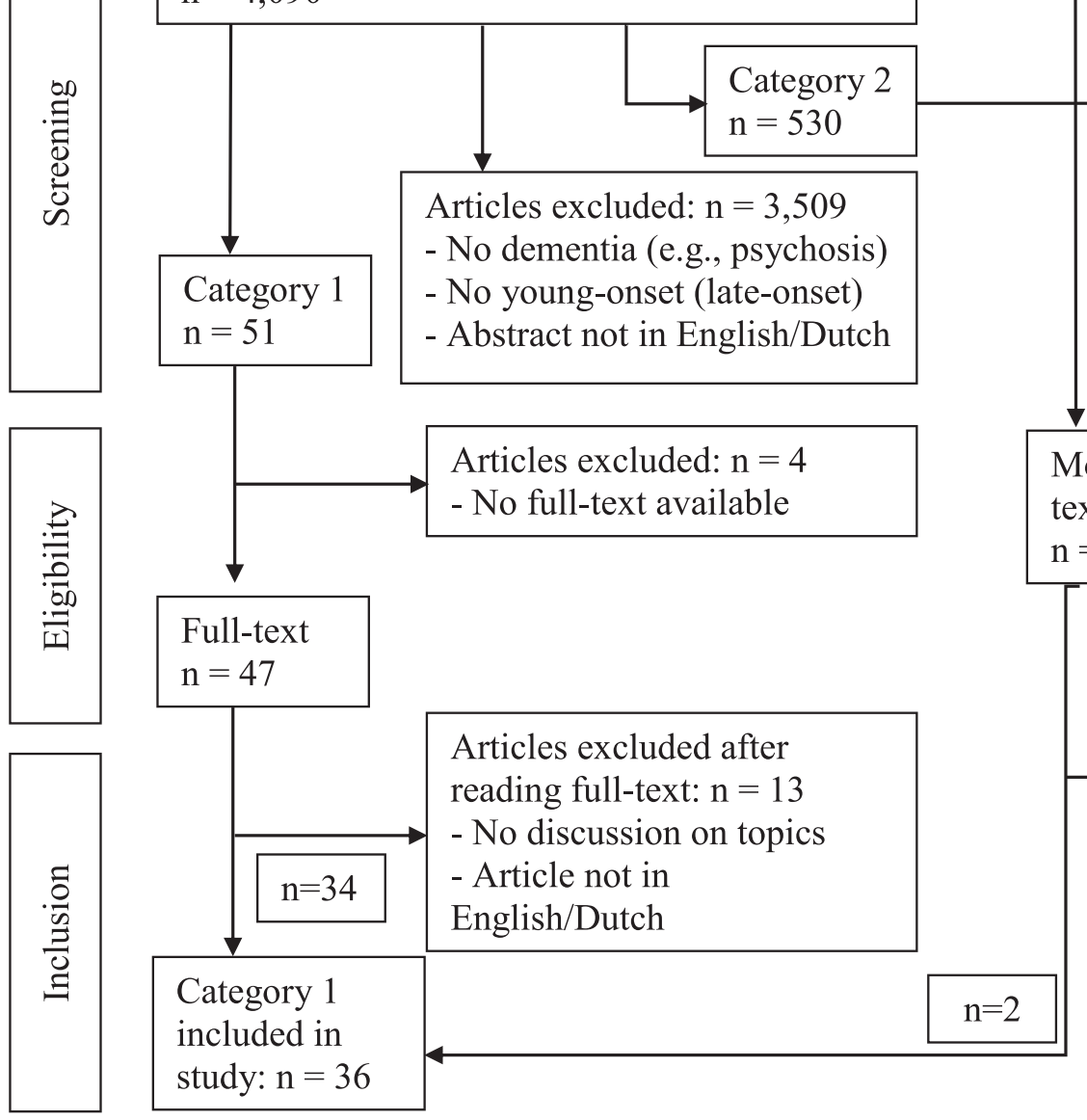

Documents

excluded:

$\mathrm{n}=98$

- No new

information

\section{Category 2}

$10 \%$ sample:

$\mathrm{n}=53$

Fig. 1. Data evaluation process. 
documents were included that explicitly discussed the terminology, definition, or both of dementia at a young age and that provided new information. Other documents included local or national dementia policies or strategies and PDF files of PowerPoint presentations; most without any discussion regarding dementia at a young age. Reading the next selection of 50 theoretical studies did not provide any new information. A manual reference list check of the included category 1 articles did not yield new articles. Furthermore, a total of 530 category 2 empirical studies were found that included the terminology, definition, or both of dementia at a young age, without explicitly discussing these aspects. A sample of 53 articles was included. Altogether, this resulted in the inclusion of 89 sources.

\section{Quality assessment}

A total of 29 publications were appraised as good, including both empirical and theoretical literature, whereas seven publications were appraised as adequate, primarily due to limitations regarding the methodological quality or representativity (Table 1). None of the included sources were appraised as inadequate.

\section{General findings}

The majority of the included literature consisted of discussion papers and editorials supplemented with 16 reviews, one quantitative study, and two other documents that addressed the points of interest in this review. A total of seven different terms, six upper age limits, four cut-off age criteria, eight lower age limits, an option for subdivision based on age, 251 different etiologies, and 27 separate categories of etiologies were identified (Table 1).

\section{Terminology}

A total of seven different terms were identified, of which the term presenile dementia occurred most often (i.e., 18 times, see Table 3), followed by earlyonset dementia (16), young-onset dementia (15), and younger-onset dementia (3). Young-onset dementia was the most common term in articles published in the last two decades to refer to young people living with dementia. In these studies, it is noteworthy that the term young-onset dementia or early-onset dementia was often compared to presenile dementia, in which the latter term was referred to as outdated [25] and last used over a decade ago [26]. This finding was supported after verification with a sample of category 2 literature (Table 2), in which the term presenile dementia was last used in empirical literature in 2004 [27]. The term early-onset dementia was commonly used until 2013 [28], after which the term was not found to be the preferred term in discussions about the terminology of dementia at a young age. However, this term was used in 'category 2 literature' after 2013. The term young-onset dementia first appeared around the new millennium, both in theoretical literature [29] and in empirical literature [5] and is still commonly used. In addition to a historical evolution in the use of terminology, some authors have suggested that differences may exist within the group of younger people living with dementia. In their opinion, presenile dementia may typically be used to refer to causes of dementia that more exclusively occur in young individuals, whereas early-onset dementia might refer to old-age causes that also occur in young people [3]. Other authors have suggested that youngonset dementia should be used to refer to both of these types of causes, including late-onset forms of childhood neurodegenerative conditions [4]. Masellis and colleagues [28] used the term early-onset dementia to refer to people living with dementia below the age of 65 years and young-onset dementia below the age of 45 years. The term early-onset dementia could furthermore be mistaken as meaning an early stage of dementia [28]. In Australia, the term youngeronset dementia was often used and appears to be used interchangeably with the term young-onset dementia [30-32]. Other terms were only mentioned in one publication and included childhood dementia, adultonset dementia, and working-age dementia, which refer to disorders causing dementia at childhood, in young adulthood [33], and before the age of retirement, respectively [34].

\section{Age-related aspects}

To distinguish young people living with dementia from individuals who live with late-onset dementia, a cut-off age was defined in nearly all literature. In the majority of both the category 1 and the sample of category 2 literature, the age of 65 was regarded as the upper age limit (Table 3). Other upper age limits that were mentioned included the ages of $45[6,28,35], 50$ [36], 55 [36], 60 [5, 26, 29, 37-39], and 70 years [29]. The age of 45 years as an upper age limit was used as a comparison to the age of 65 to gain insight into the differences in etiologies between people aged below 
Table 2

Random sample category 2 terminology and definitions of dementia at a young age

\begin{tabular}{|c|c|c|c|c|c|c|c|c|c|}
\hline \multirow[t]{2}{*}{ Authors (y) } & \multicolumn{3}{|c|}{ Study } & \multirow[t]{2}{*}{ Term(s) } & \multicolumn{3}{|c|}{ Age-related aspects } & \multicolumn{2}{|c|}{ Etiologies $\dagger$} \\
\hline & Design & $\begin{array}{l}\text { Population } \\
\text { (n) }\end{array}$ & Setting & & $\begin{array}{l}\text { Upper age } \\
\text { limit }\end{array}$ & $\begin{array}{l}\text { Lower age } \\
\text { limit }\end{array}$ & Subdivision & Diseases & Categories \\
\hline $\begin{array}{l}\text { "Spotting } \\
\text { early onset } \\
\text { dementia" } \\
(2016) \text { [73] }\end{array}$ & Announcement & $\mathrm{n} / \mathrm{a}$ & $\mathrm{n} / \mathrm{a}$ & $\begin{array}{l}\text { Early onset } \\
\text { dementia }\end{array}$ & 65 & $\mathrm{n} / \mathrm{a}$ & $\mathrm{n} / \mathrm{a}$ & $\mathrm{n} / \mathrm{a}$ & $\mathrm{n} / \mathrm{a}$ \\
\hline $\begin{array}{l}\text { Allen and } \\
\text { Baldwin } \\
(1995) \text { [54] }\end{array}$ & $\begin{array}{l}\text { Case-control } \\
\text { study }\end{array}$ & 28 & Home & $\begin{array}{l}\text { Presenile } \\
\text { dementia }\end{array}$ & 65 & $\mathrm{n} / \mathrm{a}$ & $\mathrm{n} / \mathrm{a}$ & $\begin{array}{l}1,2,31,73 \\
\text { Exclusion: } 2 b \text {, } \\
5,8,39,81,99 \text {, } \\
145,147,169\end{array}$ & $\begin{array}{l}\mathrm{n} / \mathrm{a} \\
\text { Exclusion: } \\
\mathrm{IXb}, \text { XIIb, } \\
\text { IXb }\end{array}$ \\
\hline $\begin{array}{l}\text { Andrew et al. } \\
\text { (2019) [74] }\end{array}$ & Review & $\mathrm{n} / \mathrm{a}$ & $\mathrm{n} / \mathrm{a}$ & $\begin{array}{l}\text { Younger onset } \\
\text { dementia }\end{array}$ & 65 & $\mathrm{n} / \mathrm{a}$ & $\mathrm{n} / \mathrm{a}$ & $\mathrm{n} / \mathrm{a}$ & $\mathrm{n} / \mathrm{a}$ \\
\hline $\begin{array}{l}\text { Appelhof et al. } \\
(2019) \text { [55] }\end{array}$ & $\begin{array}{l}\text { Randomized } \\
\text { controlled } \\
\text { trial }\end{array}$ & 274 & $\begin{array}{l}\text { Nursing } \\
\text { home }\end{array}$ & $\begin{array}{l}\text { Young-onset } \\
\text { dementia }\end{array}$ & 65 & $\mathrm{n} / \mathrm{a}$ & $\mathrm{n} / \mathrm{a}$ & $\begin{array}{l}1,2,3,4,29,73 \\
\text { Exclusion: } 8, \\
57,73 b, 134, \\
161\end{array}$ & $\mathrm{n} / \mathrm{a}$ \\
\hline $\begin{array}{l}\text { Armari et al. } \\
\text { (2013) [75] }\end{array}$ & $\begin{array}{l}\text { Case-control } \\
\text { study }\end{array}$ & 83 & Home & $\begin{array}{l}\text { Early onset } \\
\text { dementia }\end{array}$ & 65 & 45 & $\mathrm{n} / \mathrm{a}$ & 1,2 & $\mathrm{n} / \mathrm{a}$ \\
\hline $\begin{array}{l}\text { Arvanitakis } \\
\text { et al. (2019) } \\
\text { [76] }\end{array}$ & Review & $\mathrm{n} / \mathrm{a}$ & $\mathrm{n} / \mathrm{a}$ & $\begin{array}{l}\text { Young onset } \\
\text { dementia }\end{array}$ & 65 & $\mathrm{n} / \mathrm{a}$ & $\mathrm{n} / \mathrm{a}$ & $1,2,3,29$ & $\mathrm{n} / \mathrm{a}$ \\
\hline $\begin{array}{l}\text { Balasa et al. } \\
\text { (2014) [77] }\end{array}$ & Cohort study & 157 & Home & $\begin{array}{l}\text { Early-onset } \\
\text { dementia }\end{array}$ & $\begin{array}{l}65 \text { at } \\
\text { diagnosis }\end{array}$ & $\mathrm{n} / \mathrm{a}$ & $\mathrm{n} / \mathrm{a}$ & $1,1 \mathrm{a}, 2,2 \mathrm{c}$ & XIIb \\
\hline $\begin{array}{l}\text { Belhouane } \\
\text { et al. (2012) } \\
\text { [78] }\end{array}$ & $\begin{array}{l}\text { Cross } \\
\text { sectional } \\
\text { study }\end{array}$ & 1685 & Hospital & $\begin{array}{l}\text { Early onset } \\
\text { dementia }\end{array}$ & $\begin{array}{l}65 \text { at } \\
\text { symptom } \\
\text { onset }\end{array}$ & $\mathrm{n} / \mathrm{a}$ & $\mathrm{n} / \mathrm{a}$ & 1,29 & I, III, Vb \\
\hline $\begin{array}{l}\text { Carter et al. } \\
\text { (2018) [18] }\end{array}$ & Review & $\mathrm{n} / \mathrm{a}$ & $\mathrm{n} / \mathrm{a}$ & $\begin{array}{l}\text { Young-onset } \\
\text { dementia }\end{array}$ & $\begin{array}{l}65 \text { at } \\
\text { symptom } \\
\text { onset }\end{array}$ & $\mathrm{n} / \mathrm{a}$ & $\mathrm{n} / \mathrm{a}$ & $1,2,2 \mathrm{a}$ & $\mathrm{n} / \mathrm{a}$ \\
\hline $\begin{array}{l}\text { Cations et al. } \\
\text { (2019) [79] }\end{array}$ & Review & $\mathrm{n} / \mathrm{a}$ & $\mathrm{n} / \mathrm{a}$ & $\begin{array}{l}\text { Young onset } \\
\text { dementia }\end{array}$ & $\begin{array}{l}60,65 \text { at } \\
\text { symptom } \\
\text { onset }\end{array}$ & $\mathrm{n} / \mathrm{a}$ & $\begin{array}{l}<45 \\
45-65\end{array}$ & $1,29,73,134,145$ & $\mathrm{n} / \mathrm{a}$ \\
\hline $\begin{array}{l}\text { Dale (2000) } \\
\text { [80] }\end{array}$ & Comment & $\mathrm{n} / \mathrm{a}$ & $\mathrm{n} / \mathrm{a}$ & $\begin{array}{l}\text { Presenile } \\
\text { dementia }\end{array}$ & $\mathrm{n} / \mathrm{a}$ & $\mathrm{n} / \mathrm{a}$ & $\mathrm{n} / \mathrm{a}$ & 1,2 & $\mathrm{n} / \mathrm{a}$ \\
\hline $\begin{array}{l}\text { Devineni and } \\
\text { Onyike } \\
(2015)[81]\end{array}$ & Review & $\mathrm{n} / \mathrm{a}$ & $\mathrm{n} / \mathrm{a}$ & $\begin{array}{l}\text { Young-onset } \\
\text { dementia, } \\
\text { early-onset } \\
\text { dementia, } \\
\text { presenile } \\
\text { dementia }\end{array}$ & 65,45 & $\begin{array}{l}17,20,35, \\
45\end{array}$ & $\mathrm{n} / \mathrm{a}$ & $\begin{array}{l}1,1 \mathrm{a}, 2,2 \mathrm{a}, 2 \mathrm{c}, \\
\text { 2c-i, } 2 \mathrm{c}-\mathrm{ii}, \\
\text { 2c-iii, } 3,4,5,6, \\
8,9,14,29,58 \mathrm{a}, \\
73,134\end{array}$ & $\begin{array}{l}\text { I, II, IVa, IV, } \\
\text { Vb, Vc, } \\
\text { XIII, XV }\end{array}$ \\
\hline
\end{tabular}


Table 2

(Continued)

\begin{tabular}{|c|c|c|c|c|c|c|c|c|c|}
\hline \multirow[t]{2}{*}{ Authors (y) } & \multicolumn{3}{|c|}{ Study } & \multirow[t]{2}{*}{ Term(s) } & \multicolumn{3}{|c|}{ Age-related aspects } & \multicolumn{2}{|c|}{ Etiologies $\dagger$} \\
\hline & Design & $\begin{array}{l}\text { Population } \\
\text { (n) }\end{array}$ & Setting & & $\begin{array}{l}\text { Upper age } \\
\text { limit }\end{array}$ & $\begin{array}{l}\text { Lower age } \\
\text { limit }\end{array}$ & Subdivision & Diseases & Categories \\
\hline $\begin{array}{l}\text { Diehl-Schmid } \\
\text { et al. (2018) } \\
\text { [82] }\end{array}$ & Protocol paper & $\mathrm{n} / \mathrm{a}$ & $\mathrm{n} / \mathrm{a}$ & $\begin{array}{l}\text { Young-onset } \\
\text { dementia }\end{array}$ & $\begin{array}{l}65 \text { at } \\
\text { symptom } \\
\text { onset }\end{array}$ & $\mathrm{n} / \mathrm{a}$ & $\mathrm{n} / \mathrm{a}$ & $\mathrm{n} / \mathrm{a}$ & $\mathrm{n} / \mathrm{a}$ \\
\hline $\begin{array}{l}\text { Draper et al. } \\
\text { (2016) [62] }\end{array}$ & Cohort study & 88 & Home & $\begin{array}{l}\text { Young-onset } \\
\text { dementia }\end{array}$ & $\begin{array}{l}65 \text { at } \\
\text { symptom } \\
\text { onset }\end{array}$ & $\mathrm{n} / \mathrm{a}$ & $\mathrm{n} / \mathrm{a}$ & $1,2,3,5,8,29,73$ & $\mathrm{n} / \mathrm{a}$ \\
\hline $\begin{array}{l}\text { Dumitru et al. } \\
\text { (2014) [83] }\end{array}$ & $\begin{array}{l}\text { Cross } \\
\text { sectional } \\
\text { study }\end{array}$ & 350 & Hospital & $\begin{array}{l}\text { Early-onset } \\
\text { dementia }\end{array}$ & 65 & $\mathrm{n} / \mathrm{a}$ & $\mathrm{n} / \mathrm{a}$ & $1,2,29,73,134$ & $\mathrm{n} / \mathrm{a}$ \\
\hline $\begin{array}{l}\text { Filipovic and } \\
\text { Kostic } \\
\text { (1995) [84] }\end{array}$ & $\begin{array}{l}\text { Randomized } \\
\text { controlled } \\
\text { trial }\end{array}$ & 137 & Home & $\begin{array}{l}\text { Presenile } \\
\text { dementia }\end{array}$ & 65 & $\mathrm{n} / \mathrm{a}$ & $\mathrm{n} / \mathrm{a}$ & $1,4,8,31$ & $\mathrm{n} / \mathrm{a}$ \\
\hline $\begin{array}{l}\text { Fujihara et al. } \\
\text { (2004) [27] }\end{array}$ & $\begin{array}{l}\text { Cross } \\
\text { sectional } \\
\text { study }\end{array}$ & 141 & Hospital & $\begin{array}{l}\text { Presenile } \\
\text { dementia, } \\
\text { early-onset } \\
\text { dementia }\end{array}$ & $\begin{array}{l}65 \text { at } \\
\text { symptom } \\
\text { onset }\end{array}$ & 21 & $\mathrm{n} / \mathrm{a}$ & $\begin{array}{l}1,2,4,5,29,39 \\
43,49,70,73 \\
121,134,135 \\
162,169\end{array}$ & $\mathrm{Vb}$ \\
\hline $\begin{array}{l}\text { Garre-Olmo } \\
\text { et al. }(2010) \\
{[85]}\end{array}$ & $\begin{array}{l}\text { Cross } \\
\text { sectional } \\
\text { study }\end{array}$ & 2083 & Hospital & $\begin{array}{l}\text { Early-onset } \\
\text { dementia }\end{array}$ & $\begin{array}{l}65 \text { at } \\
\text { symptom } \\
\text { onset }\end{array}$ & 30 & $\mathrm{n} / \mathrm{a}$ & $\begin{array}{l}1,2,2 \mathrm{a}, 2 \mathrm{c}-\mathrm{i}, 3,4 \\
5,6,8,29,58 \mathrm{a} \\
73,134\end{array}$ & $\mathrm{n} / \mathrm{a}$ \\
\hline $\begin{array}{l}\text { Giebel et al. } \\
\text { (2020) [86] }\end{array}$ & Cohort study & 11 & Home & $\begin{array}{l}\text { Young-onset } \\
\text { dementia }\end{array}$ & $\begin{array}{l}65 \text { at } \\
\text { diagnosis }\end{array}$ & $\mathrm{n} / \mathrm{a}$ & $\mathrm{n} / \mathrm{a}$ & $1,2,29$ & $\mathrm{n} / \mathrm{a}$ \\
\hline $\begin{array}{l}\text { Gordon (1968) } \\
\text { [87] }\end{array}$ & Cohort study & 13 & Home & $\begin{array}{l}\text { Presenile } \\
\text { dementia }\end{array}$ & $\mathrm{n} / \mathrm{a}$ & $\mathrm{n} / \mathrm{a}$ & $\mathrm{n} / \mathrm{a}$ & $1,2,58 \mathrm{a}$ & $\mathrm{n} / \mathrm{a}$ \\
\hline $\begin{array}{l}\text { Gottfries et al. } \\
\text { (1969) [88] }\end{array}$ & Cohort study & 77 & Home & $\begin{array}{l}\text { Presenile } \\
\text { dementia }\end{array}$ & $\mathrm{n} / \mathrm{a}$ & $\mathrm{n} / \mathrm{a}$ & $\mathrm{n} / \mathrm{a}$ & $\mathrm{n} / \mathrm{a}$ & $\mathrm{n} / \mathrm{a}$ \\
\hline $\begin{array}{l}\text { Guermani } \\
\text { et al. (2013) } \\
\text { [89] }\end{array}$ & $\begin{array}{l}\text { Cross } \\
\text { sectional } \\
\text { study }\end{array}$ & 50 & Hospital & $\begin{array}{l}\text { Early onset } \\
\text { dementia }\end{array}$ & $\mathrm{n} / \mathrm{a}$ & $\mathrm{n} / \mathrm{a}$ & $\mathrm{n} / \mathrm{a}$ & $\mathrm{n} / \mathrm{a}$ & $\mathrm{n} / \mathrm{a}$ \\
\hline $\begin{array}{l}\text { Gustafson and } \\
\text { Nilsson } \\
\text { (1982) [90] }\end{array}$ & Cohort study & 57 & Home & $\begin{array}{l}\text { Presenile } \\
\text { dementia }\end{array}$ & 65 & 40 & $\mathrm{n} / \mathrm{a}$ & $\begin{array}{l}1,2,31,49,58 \mathrm{a}, \\
135\end{array}$ & I, II \\
\hline $\begin{array}{l}\text { Harvey et al. } \\
(2003)[46]\end{array}$ & $\begin{array}{l}\text { Cross } \\
\text { sectional } \\
\text { study } \\
\end{array}$ & 227 & Hospital & $\begin{array}{l}\text { Young-onset } \\
\text { dementia }\end{array}$ & 65 & 30 & $\begin{array}{l}30-44 \\
45-64\end{array}$ & $\begin{array}{l}1,2,3,4,6,8,29 \\
39,73,161\end{array}$ & IVa \\
\hline
\end{tabular}




\begin{tabular}{|c|c|c|c|c|c|c|c|c|c|}
\hline $\begin{array}{l}\text { Henley et al. } \\
\text { (2014) [91] }\end{array}$ & $\begin{array}{l}\text { Project } \\
\text { description }\end{array}$ & $\mathrm{n} / \mathrm{a}$ & $\mathrm{n} / \mathrm{a}$ & $\begin{array}{l}\text { Young-onset } \\
\text { dementia, } \\
\text { early-onset } \\
\text { dementia }\end{array}$ & $\begin{array}{l}65 \text { at } \\
\text { diagnosis }\end{array}$ & $\mathrm{n} / \mathrm{a}$ & $\mathrm{n} / \mathrm{a}$ & $\begin{array}{l}\text { 1, 1a, 2, 2a, 2c, 3, } \\
4,5,8\end{array}$ & $\mathrm{n} / \mathrm{a}$ \\
\hline $\begin{array}{l}\text { Hutchinson } \\
\text { et al. (2016) } \\
\text { [92] }\end{array}$ & Cohort study & 12 & $\begin{array}{l}\text { Home and } \\
\text { nursing } \\
\text { home }\end{array}$ & $\begin{array}{l}\text { Younger-onset } \\
\text { dementia }\end{array}$ & 65 & 30 & $\mathrm{n} / \mathrm{a}$ & 1 & $\mathrm{n} / \mathrm{a}$ \\
\hline $\begin{array}{l}\text { Hvidsten et al. } \\
\text { (2019) [93] }\end{array}$ & Cohort study & 88 & $\begin{array}{l}\text { Nursing } \\
\text { home }\end{array}$ & $\begin{array}{l}\text { Young-onset } \\
\text { dementia }\end{array}$ & $\begin{array}{l}65 \text { at } \\
\text { symptom } \\
\text { onset }\end{array}$ & $\mathrm{n} / \mathrm{a}$ & $\mathrm{n} / \mathrm{a}$ & $1,2,2 \mathrm{a}, 2 \mathrm{c}$ & $\mathrm{n} / \mathrm{a}$ \\
\hline $\begin{array}{l}\text { Ikejima et al. } \\
\text { (2009) [58] }\end{array}$ & $\begin{array}{l}\text { Cross } \\
\text { sectional } \\
\text { study }\end{array}$ & 617 & Hospital & $\begin{array}{l}\text { Early-onset } \\
\text { dementia, } \\
\text { presenile } \\
\text { dementia }\end{array}$ & $\begin{array}{l}65 \text { at } \\
\text { symptom } \\
\text { onset }\end{array}$ & 20 & $\mathrm{n} / \mathrm{a}$ & $\begin{array}{l}1,3,4,29,30,31, \\
33,73,133, \\
134,169 \\
\text { Exclusion: } 162, \\
166\end{array}$ & $\begin{array}{l}\text { IV } \\
\text { Exclusion: } \\
\text { XIIa }\end{array}$ \\
\hline $\begin{array}{l}\text { Jefferies and } \\
\text { Agrawal } \\
(2009) \text { [56] }\end{array}$ & Review & $\mathrm{n} / \mathrm{a}$ & $\mathrm{n} / \mathrm{a}$ & $\begin{array}{l}\text { Early-onset } \\
\text { dementia, } \\
\text { young-onset } \\
\text { dementia }\end{array}$ & 65 & 30 & $\mathrm{n} / \mathrm{a}$ & $\begin{array}{l}\text { 1, 2, 2a, 2c, 2c-i, } \\
3,8,29,29 \mathrm{a}, 30 \text {, } \\
31,33,57,58 \mathrm{a} \text {, } \\
73 \\
\text { Exclusion: } 39 \text {, } \\
\text { 43, 43b, 45, 61a, } \\
73 \mathrm{~b}, 73 \mathrm{f}, 77, \\
\text { 134, 135, 162, } \\
\text { 169, delirium, } \\
\text { amnestic } \\
\text { syndromes, } \\
\text { MCI, pseudode- } \\
\text { mentia, stroke, } \\
\text { encephalitis, } \\
\text { dissociative } \\
\text { disorders, } \\
\text { benzodiazepines }\end{array}$ & $\begin{array}{l}\text { IVa } \\
\text { Exclusion: } \\
\text { XI }\end{array}$ \\
\hline $\begin{array}{l}\text { Johannessen } \\
\text { et al. (2014) } \\
\text { [94] }\end{array}$ & $\begin{array}{l}\text { Cross } \\
\text { sectional } \\
\text { study }\end{array}$ & 20 & Home & $\begin{array}{l}\text { Young onset } \\
\text { dementia }\end{array}$ & $\begin{array}{l}65 \text { at } \\
\text { diagnosis }\end{array}$ & $\mathrm{n} / \mathrm{a}$ & $\mathrm{n} / \mathrm{a}$ & 1 & $\mathrm{n} / \mathrm{a}$ \\
\hline $\begin{array}{l}\text { Kaszniak et al. } \\
\text { (1978) [95] }\end{array}$ & Cohort study & 47 & $\begin{array}{l}\text { Nursing } \\
\text { home }\end{array}$ & $\begin{array}{l}\text { Presenile } \\
\text { dementia }\end{array}$ & $\mathrm{n} / \mathrm{a}$ & $\mathrm{n} / \mathrm{a}$ & $\mathrm{n} / \mathrm{a}$ & $\mathrm{n} / \mathrm{a}$ & $\mathrm{n} / \mathrm{a}$ \\
\hline
\end{tabular}


Table 2

(Continued)

\begin{tabular}{|c|c|c|c|c|c|c|c|c|c|}
\hline \multirow[t]{2}{*}{ Authors (y) } & \multicolumn{3}{|c|}{ Study } & \multirow{2}{*}{ Term(s) } & \multicolumn{3}{|c|}{ Age-related aspects } & \multicolumn{2}{|c|}{ Etiologies $\dagger$} \\
\hline & Design & $\begin{array}{l}\text { Population } \\
\text { (n) }\end{array}$ & Setting & & $\begin{array}{l}\text { Upper age } \\
\text { limit }\end{array}$ & $\begin{array}{l}\text { Lower age } \\
\text { limit }\end{array}$ & Subdivision & Diseases & Categories \\
\hline $\begin{array}{l}\text { Kelley et al. } \\
(2008) \text { [40] }\end{array}$ & $\begin{array}{l}\text { Cross } \\
\text { sectional } \\
\text { study }\end{array}$ & 235 & Hospital & $\begin{array}{l}\text { Young-onset } \\
\text { dementia, early } \\
\text { onset dementia }\end{array}$ & $\begin{array}{l}65,45 \text { at } \\
\text { symptom } \\
\text { onset }\end{array}$ & $17,21,30$ & $\begin{array}{l}30-44, \\
45-65\end{array}$ & $\begin{array}{l}\text { 1, 1a, 2, 2d, 3, } 4 \\
5,6,8,10,12, \\
14,17,25,29, \\
30,31,39,43, \\
43 \mathrm{~b}, 50,57,71, \\
73,87,108, \\
110,112,120, \\
121,123,129, \\
133,135,142\end{array}$ & $\begin{array}{l}\text { I, II, III, IV, } \\
\text { IVa, Va, Vb, } \\
\text { VI, VII, X, } \\
\text { XIV }\end{array}$ \\
\hline $\begin{array}{l}\text { Kilty et al. } \\
\text { (2019) [96] }\end{array}$ & $\begin{array}{l}\text { Cross } \\
\text { sectional } \\
\text { study }\end{array}$ & 6 & $\begin{array}{l}\text { Home and } \\
\text { nursing } \\
\text { home }\end{array}$ & $\begin{array}{l}\text { Young onset } \\
\text { dementia }\end{array}$ & 65 & $\mathrm{n} / \mathrm{a}$ & $\mathrm{n} / \mathrm{a}$ & $\mathrm{n} / \mathrm{a}$ & $\mathrm{n} / \mathrm{a}$ \\
\hline $\begin{array}{l}\text { Koopmans } \\
\text { et al. }(2015) \\
{[97]}\end{array}$ & Editorial & $\mathrm{n} / \mathrm{a}$ & $\mathrm{n} / \mathrm{a}$ & $\begin{array}{l}\text { Young-onset } \\
\text { dementia }\end{array}$ & $\begin{array}{l}65 \text { at } \\
\text { symptom } \\
\text { onset }\end{array}$ & $\mathrm{n} / \mathrm{a}$ & $\begin{array}{l}30-44 \\
45-65\end{array}$ & 1 & $\mathrm{n} / \mathrm{a}$ \\
\hline $\begin{array}{l}\text { Kurz et al. } \\
\text { (2016) [98] }\end{array}$ & Protocol paper & $\mathrm{n} / \mathrm{a}$ & $\mathrm{n} / \mathrm{a}$ & $\begin{array}{l}\text { Young-onset } \\
\text { dementia }\end{array}$ & $\begin{array}{l}65 \text { at } \\
\text { symptom } \\
\text { onset }\end{array}$ & $\mathrm{n} / \mathrm{a}$ & $\mathrm{n} / \mathrm{a}$ & 1 & $\mathrm{n} / \mathrm{a}$ \\
\hline $\begin{array}{l}\text { Liddell (1958) } \\
\text { [99] }\end{array}$ & Cohort study & 11 & Hospital & $\begin{array}{l}\text { Presenile } \\
\text { dementia }\end{array}$ & $\mathrm{n} / \mathrm{a}$ & $\mathrm{n} / \mathrm{a}$ & $\mathrm{n} / \mathrm{a}$ & $\mathrm{n} / \mathrm{a}$ & $\mathrm{n} / \mathrm{a}$ \\
\hline $\begin{array}{l}\text { Maiovis et al. } \\
(2015) \text { [100] }\end{array}$ & $\begin{array}{l}\text { Cross } \\
\text { sectional } \\
\text { study }\end{array}$ & 491 & Hospital & $\begin{array}{l}\text { Early onset } \\
\text { dementia }\end{array}$ & $\begin{array}{l}65 \text { at } \\
\text { symptom } \\
\text { onset }\end{array}$ & $\mathrm{n} / \mathrm{a}$ & $\mathrm{n} / \mathrm{a}$ & $\begin{array}{l}1,2,2 \mathrm{a}, 2 \mathrm{c}-\mathrm{i} \\
2 \mathrm{c}-\mathrm{ii}, 3,5,6\end{array}$ & $\begin{array}{l}\text { I, II, III, IV, } \\
\text { Va, Vb, IXc, } \\
\text { XIII }\end{array}$ \\
\hline $\begin{array}{l}\text { McMurtray } \\
\text { et al. }(2006) \\
{[101]}\end{array}$ & $\begin{array}{l}\text { Cross } \\
\text { sectional } \\
\text { study }\end{array}$ & 1683 & Hospital & $\begin{array}{l}\text { Early-onset } \\
\text { dementia }\end{array}$ & $\begin{array}{l}65 \text { at } \\
\text { symptom } \\
\text { onset }\end{array}$ & $\mathrm{n} / \mathrm{a}$ & $\begin{array}{l}<45 \\
45-65\end{array}$ & $\begin{array}{l}1,2,3,4,6,7,8 \\
29,39,57,58 \mathrm{a} \\
60,73,73 \mathrm{c}, 86 \\
134,135\end{array}$ & $\mathrm{n} / \mathrm{a}$ \\
\hline $\begin{array}{l}\text { Nordstrom } \\
\text { et al. }(2013) \\
\text { [57] }\end{array}$ & $\begin{array}{l}\text { Case-control } \\
\text { study }\end{array}$ & 497844 & Hospital & $\begin{array}{l}\text { Young-onset } \\
\text { dementia, } \\
\text { early-onset } \\
\text { dementia }\end{array}$ & 65 & $\mathrm{n} / \mathrm{a}$ & $\mathrm{n} / \mathrm{a}$ & $\begin{array}{l}1,3,4,29,73 \\
\text { Exclusion: } 162, \\
\text { stroke, } \\
\text { myocardinfarct }\end{array}$ & $\begin{array}{l}\mathrm{n} / \mathrm{a} \\
\text { Exclusion: } \\
\mathrm{Va}\end{array}$ \\
\hline $\begin{array}{l}\text { Phuapradit } \\
\text { et al. (1978) } \\
\text { [102] }\end{array}$ & Comment & $\mathrm{n} / \mathrm{a}$ & $\mathrm{n} / \mathrm{a}$ & $\begin{array}{l}\text { Presenile } \\
\text { dementia }\end{array}$ & $\mathrm{n} / \mathrm{a}$ & $\mathrm{n} / \mathrm{a}$ & $\mathrm{n} / \mathrm{a}$ & 1 & $\mathrm{n} / \mathrm{a}$ \\
\hline $\begin{array}{l}\text { Rizzo et al. } \\
\text { (1984) [103] }\end{array}$ & $\begin{array}{l}\text { Cross } \\
\text { sectional } \\
\text { study }\end{array}$ & 8 & Hospital & $\begin{array}{l}\text { Presenile } \\
\text { dementia }\end{array}$ & $\mathrm{n} / \mathrm{a}$ & $\mathrm{n} / \mathrm{a}$ & $\mathrm{n} / \mathrm{a}$ & $\mathrm{n} / \mathrm{a}$ & $\mathrm{n} / \mathrm{a}$ \\
\hline $\begin{array}{l}\text { Rogers and } \\
\text { Lippa } \\
\text { (2012) [104] }\end{array}$ & Review & $\mathrm{n} / \mathrm{a}$ & $\mathrm{n} / \mathrm{a}$ & $\begin{array}{l}\text { Early-onset } \\
\text { dementia }\end{array}$ & 65 & $\mathrm{n} / \mathrm{a}$ & $\mathrm{n} / \mathrm{a}$ & $\begin{array}{l}1,2,2 \mathrm{a}, 2 \mathrm{c}-\mathrm{i} \\
2 \mathrm{c}-\mathrm{ii}, 110,112\end{array}$ & $\mathrm{n} / \mathrm{a}$ \\
\hline
\end{tabular}




\begin{tabular}{|c|c|c|c|c|c|c|c|c|c|}
\hline $\begin{array}{l}\text { Ron et al. } \\
\text { (1979) [59] }\end{array}$ & $\begin{array}{l}\text { Cross } \\
\text { sectional } \\
\text { study }\end{array}$ & 52 & Hospital & $\begin{array}{l}\text { Presenile } \\
\text { dementia }\end{array}$ & $\begin{array}{l}65 \text { at } \\
\text { diagnosis }\end{array}$ & $\mathrm{n} / \mathrm{a}$ & $\mathrm{n} / \mathrm{a}$ & $\begin{array}{l}\text { n/a } \\
\text { Exclusion: } 73 \text {, } \\
77,134,147, \\
\text { space- } \\
\text { occupying } \\
\text { lesion, } \\
\text { intracranial } \\
\text { infection }\end{array}$ & $\begin{array}{l}\mathrm{n} / \mathrm{a} \\
\text { Exclusion: } \\
\mathrm{II}, \mathrm{Vb}, \mathrm{XI}\end{array}$ \\
\hline $\begin{array}{l}\text { Sansoni et al. } \\
\text { (2016) [105] }\end{array}$ & Review & $\mathrm{n} / \mathrm{a}$ & $\mathrm{n} / \mathrm{a}$ & $\begin{array}{l}\text { Younger onset } \\
\text { dementia, early } \\
\text { onset dementia }\end{array}$ & $\begin{array}{l}60,65 \text { at } \\
\text { diagnosis }\end{array}$ & $\mathrm{n} / \mathrm{a}$ & $\mathrm{n} / \mathrm{a}$ & $\begin{array}{l}1,2,3,4,8,29 \\
57,73,161\end{array}$ & $\mathrm{n} / \mathrm{a}$ \\
\hline $\begin{array}{l}\text { Smith et al. } \\
\text { (1966) [106] }\end{array}$ & $\begin{array}{l}\text { Cross } \\
\text { sectional } \\
\text { study }\end{array}$ & 59 & Hospital & $\begin{array}{l}\text { Presenile } \\
\text { dementia }\end{array}$ & $\begin{array}{l}65 \text { at } \\
\text { symptom } \\
\text { onset }\end{array}$ & $\mathrm{n} / \mathrm{a}$ & $\mathrm{n} / \mathrm{a}$ & $1,2,29,41,58$ & $\mathrm{n} / \mathrm{a}$ \\
\hline $\begin{array}{l}\text { Spreadbury } \\
\text { and Kipps } \\
\text { (2019) [107] }\end{array}$ & Review & $\mathrm{n} / \mathrm{a}$ & $\mathrm{n} / \mathrm{a}$ & $\begin{array}{l}\text { Presenile } \\
\text { dementia, } \\
\text { young onset } \\
\text { dementia, } \\
\text { younger onset } \\
\text { dementia }\end{array}$ & 65 & $\mathrm{n} / \mathrm{a}$ & $\mathrm{n} / \mathrm{a}$ & $\begin{array}{l}1,1 \mathrm{a}, 2,2 \mathrm{a}, 2 \mathrm{c}, \\
2 \mathrm{c}-\mathrm{i}, 2 \mathrm{c}-\mathrm{ii}, \\
2 \mathrm{c}-\mathrm{iii}, 3,4,6,8, \\
29\end{array}$ & IVa \\
\hline $\begin{array}{l}\text { Torvik (1970) } \\
\text { [108] }\end{array}$ & Review & $\mathrm{n} / \mathrm{a}$ & $\mathrm{n} / \mathrm{a}$ & $\begin{array}{l}\text { Presenile } \\
\text { dementia }\end{array}$ & $\mathrm{n} / \mathrm{a}$ & $\mathrm{n} / \mathrm{a}$ & $\mathrm{n} / \mathrm{a}$ & $\begin{array}{l}1,2,29,30,32 \\
73,58 \mathrm{a}\end{array}$ & $\mathrm{n} / \mathrm{a}$ \\
\hline $\begin{array}{l}\text { Vafeas et al. } \\
(2020) \text { [31] }\end{array}$ & $\begin{array}{l}\text { Quasi- } \\
\text { experimental } \\
\text { study }\end{array}$ & $\mathrm{n} / \mathrm{a}$ & $\mathrm{n} / \mathrm{a}$ & $\begin{array}{l}\text { Younger onset } \\
\text { dementia }\end{array}$ & $\mathrm{n} / \mathrm{a}$ & $\mathrm{n} / \mathrm{a}$ & $\mathrm{n} / \mathrm{a}$ & $\mathrm{n} / \mathrm{a}$ & $\mathrm{n} / \mathrm{a}$ \\
\hline $\begin{array}{l}\text { Vieira et al. } \\
\text { (2013) [109] }\end{array}$ & Review & $\mathrm{n} / \mathrm{a}$ & $\mathrm{n} / \mathrm{a}$ & $\begin{array}{l}\text { Early-onset } \\
\text { dementia, } \\
\text { presenile } \\
\text { dementia }\end{array}$ & $45,60,65$ & 17 & $\begin{array}{l}<45 \\
45-65\end{array}$ & $\begin{array}{l}1,2,2 \mathrm{c}-\mathrm{i}, 2 \mathrm{c}-\mathrm{ii}, 3 \\
4,8,29,31,57 \\
58 \mathrm{a}, 73,134 \\
135,161\end{array}$ & I \\
\hline $\begin{array}{l}\text { Wawrziczny } \\
\text { et al. (2018) } \\
\text { [60] }\end{array}$ & $\begin{array}{l}\text { Case-control } \\
\text { study }\end{array}$ & 150 & $\begin{array}{l}\text { Home and } \\
\text { nursing } \\
\text { home }\end{array}$ & $\begin{array}{l}\text { Early-onset } \\
\text { dementia }\end{array}$ & $\begin{array}{l}65 \text { at } \\
\text { symptom } \\
\text { onset }\end{array}$ & $\mathrm{n} / \mathrm{a}$ & $\mathrm{n} / \mathrm{a}$ & $1,2,3$ & $\mathrm{n} / \mathrm{a}$ \\
\hline $\begin{array}{l}\text { Withall (2013) } \\
\text { [30] }\end{array}$ & Editorial & $\mathrm{n} / \mathrm{a}$ & $\mathrm{n} / \mathrm{a}$ & $\begin{array}{l}\text { Younger-onset } \\
\text { dementia }\end{array}$ & $\begin{array}{l}65 \text { at } \\
\text { symptom } \\
\text { onset }\end{array}$ & 20 & $\begin{array}{l}30-45 \\
45-65\end{array}$ & $\begin{array}{l}1,2,3,4,8,39 \\
57,73,134 \\
145,161\end{array}$ & $\mathrm{n} / \mathrm{a}$ \\
\hline $\begin{array}{l}\text { Woodburn and } \\
\text { Johnstone } \\
\text { (1999) [110] }\end{array}$ & $\begin{array}{l}\text { Cross } \\
\text { sectional } \\
\text { study }\end{array}$ & 126 & $\begin{array}{l}\text { Home and } \\
\text { nursing } \\
\text { home }\end{array}$ & $\begin{array}{l}\text { Early-onset } \\
\text { dementia }\end{array}$ & $\mathrm{n} / \mathrm{a}$ & $\mathrm{n} / \mathrm{a}$ & $\mathrm{n} / \mathrm{a}$ & $1,31,73$ & $\mathrm{n} / \mathrm{a}$ \\
\hline $\begin{array}{l}\text { Wright et al. } \\
\text { (1984) [111] }\end{array}$ & Experimental & 34 & Hospital & $\begin{array}{l}\text { Presenile } \\
\text { dementia }\end{array}$ & $\begin{array}{l}70 \text { at } \\
\text { symptom } \\
\text { onset }\end{array}$ & $\mathrm{n} / \mathrm{a}$ & $\mathrm{n} / \mathrm{a}$ & $1,2,8,58 \mathrm{a}$ & $\mathrm{n} / \mathrm{a}$ \\
\hline
\end{tabular}

${ }^{\dagger}$ See Table 4. 
Table 3

Overview of different terms and age-related aspects

\begin{tabular}{|c|c|c|}
\hline Aspect & $\begin{array}{l}\text { Frequency } \\
\text { category } 1\end{array}$ & $\begin{array}{l}\text { Frequency } \\
\text { category } 2\end{array}$ \\
\hline \multicolumn{3}{|l|}{ Nomenclature } \\
\hline Presenile dementia & 18 & 20 \\
\hline Early-onset dementia & 16 & 22 \\
\hline Young-onset dementia & 15 & 19 \\
\hline Younger-onset dementia & 3 & 6 \\
\hline Adult-onset dementia & 1 & 0 \\
\hline Working-age dementia & 1 & 0 \\
\hline Childhood dementia & 1 & 0 \\
\hline \multicolumn{3}{|l|}{ Age-related aspects } \\
\hline \multicolumn{3}{|l|}{ Upper age limit } \\
\hline 45 years & 3 & 3 \\
\hline 50 years & 1 & 0 \\
\hline 55 years & 1 & 0 \\
\hline 60 years & 7 & 3 \\
\hline 65 years & 28 & 41 \\
\hline 70 years & 1 & 1 \\
\hline \multicolumn{3}{|l|}{ Age criterium } \\
\hline Age at symptom onset & 12 & 18 \\
\hline Age at diagnosis & 4 & 6 \\
\hline $\begin{array}{l}\text { Age at presentation } \\
\text { to medical services }\end{array}$ & 1 & 0 \\
\hline Age before deceased & 1 & 0 \\
\hline \multicolumn{3}{|l|}{ Lower age limit } \\
\hline $\begin{array}{l}16 \text { years } \\
\text { (incl early adulthood) }\end{array}$ & 2 & 0 \\
\hline 17 years & 1 & 3 \\
\hline 18 years & 1 & 0 \\
\hline 20 years & 1 & 3 \\
\hline 21 years & 0 & 2 \\
\hline 30 years & 4 & 5 \\
\hline 35 years & 1 & 1 \\
\hline 40 years & 4 & 1 \\
\hline 45 years & 3 & 2 \\
\hline Subdivision & 7 & 7 \\
\hline
\end{tabular}

45 and below 65 years living with dementia [40]. Other authors did not mention a reason for choosing a specific upper age limit.

In addition to a cut-off age, a distinction between age at onset or age at diagnosis was made in half of the category 1 literature. In two-thirds of this literature, age at symptom onset was mentioned, whereas in four publications, the age at diagnosis was mentioned as a criterion [29, 34, 35, 41]. A similar ratio was observed in the sample of category 2 literature, with 18 publications using age at symptom onset and six publications using age at diagnosis. None of the authors mentioned an explicit rationale to support their decision. Furthermore, two additional criteria were identified in two publications, i.e., age at 'presentation at medical services' [29], which to some extent resembles age at diagnosis, and a criterion 'died before the age of 65' [33], which was used in a postmortem study.
In one-third of all category 1 literature, the importance of distinguishing young people living with dementia from those with childhood or developmental disorders is emphasized, and a lower age limit is defined by the authors. However, in none of the publications was a reason for the use of such a lower age limit given. The way in which such a lower age limit was mentioned varied from the use of explicit ages to a more narrative description. For instance, some authors mentioned ages between 16 and 20 years $[5,6,28,35,41]$ or between 30 and 45 years [4-6, $29,39,42-45]$, while other authors more implicitly used descriptive terms such as "from early adulthood" [28], "covers the period from..." [42], and "typically afflicts patients between..." [4]. Additionally, in the sample of category 2 literature, a lower age limit of 21 years was found in two publications, although no explanation for this age limit was given. Some publications mentioned the youngest patient in their study as a lower age limit without mentioning a specific lower age limit in the methodology section [27].

Finally, seven publications described a subdivision within the group of young people living with dementia. All seven described a division into two age groups: people younger and older than 45 years. Such a subdivision was primarily used for research purposes based on the etiological differences between these two groups, e.g., a lower prevalence of Alzheimer's disease in the youngest group [32]. In one publication, the group of people aged below 45 was divided into two groups: younger and older than 35 years [46]. According to these authors, metabolic and childhood etiologies characterized the youngest group, whereas degenerative disorders caused dementia in most cases in people aged 35 years and over. In the verification process using the random sample of category 2 literature, seven publications were found that used a subdivision at the age of 45 years.

\section{Etiologies}

A total of 251 different causes of dementia were identified (Table 4). The most frequently mentioned etiologies included Alzheimer's disease (35 publications), frontotemporal dementia (30), Huntington's disease (22), alcohol-related dementias (21), and infectious diseases (26), such as human immunodeficiency virus (HIV) and prion diseases. In six literature reviews, a large variety of etiologies were discussed [4-6, 28, 41, 47]. In five of these reviews, the inclusion of an extra element in the diagnosis of dementia 
Alzheimer's disease (including: 1a posterior cortical atrophy (PCA)), 2 frontotemporal lobe degeneration (FTLD)/Pick's disease (including: 2a behavioral variant frontotemporal dementia (bvFTD)/frontal variant, $2 \mathrm{~b}$ motor neuron disease (mnFTD), 2c primary progressive aphasia (PPA), including: 2c-i semantic dementia (SD)/temporal variant, 2c-ii progressive nonfluent aphasia (PNFA), 2c-iii logopenic variant, 2d FTD and parkinsonism linked to chromosome 17 (FTDP-17), 2e dementia lacking distinctive histopathology (DLDH), 2f inclusion body myopathy associated with Paget disease and FTD (IBMPFTD)), 3 dementia with Lewy bodies (LBD), 4 Parkinson's disease dementia, 5 progressive supranuclear palsy (PSP)/Steele-Richardson-Olszewski syndrome, 6 corticobasal degeneration (CBD), 7 multiple system atrophy (MSA), 8 Huntington's disease, 9 amyotrophic lateral sclerosis (ALS), 10 pantothenate kinase-associated degeneration or neurodegeneration with iron accumulation (PKAN/NBIA/Hallervorden-Spatz syndrome), 11 aceruloplasminemia, 12 neuroacanthocystosis (McLeod's syndrome), 13a Huntington chorea-like type 1 (HDL1), 13b HDL2, 13c HDL3, 14 spinocerebellar ataxia (SCA) (including: 14a fragile X tremor ataxia syndrome (FXTAS), 14b SCA type 2, 14c SCA type 12, 14d SCA type 17), 15 familial encephalopathy with neuroserpin inclusion bodies (FENIB), 16 neuronal intermediate filament inclusion disease (NIFID)/neurofilament inclusion body disease, 17 diffuse (cortical) neurofibrillary tangle (disease) with calcification (DCNTDC/DNTC)/Kosaka-Shibayama disease/Fahr's syndrome, 18 hereditary hemochromatosis, 19 dentatorubral-pallidoluysian atrophy

(DRPLA), 20 Lafora body disease, 21 Mohr-Tranebjaerg syndrome, 22 myotonic dystrophy type 3, 23 neuroferritinopathy, 24 giant axonal neuropathy (GAN), 25 progressive myoclonic epilepsy syndrome (PME), 26 intranuclear inclusion body disease (INIBD)/neuronal intranuclear inclusion disease (NIID), 27 Perry syndrome, 28 hereditary sensory neuropathy type IE.

\section{Cerebrovascular dementias}

29 Vascular dementia (including: 29a small vessel disease (SVD), 29b large vessel disease), 30 cerebral autosomal dominant arteriopathy with subcortical infarcts and leukoencephalopathy (CADASIL)/Binswanger disease, 31 multi-infarct dementia, 32 microinfarcts, 33 strategic infarct dementia (including: 33 a thalamic dementia), 34 vascular cognitive impairment, 35 cerebral amyloid angiopathy (CAA) (including: 35a familial British dementia/English-type amyloid angiopathy, 35b familial Danish dementia, 35c Dutch variant of hereditary cerebral haemorrhage with amyloidosis, 35d hereditary cerebral haemorrhage with amyloidosis of Icelandic type, 35e meningovascular amyloidosis, 35f familial amyloidosis of Finnish type, 35g hereditary cerebral haemorrhage with amyloidosis), 36 Sneddon's syndrome, 37 antiphospholipid syndrome, 38 Susac syndrome.

\section{Inflammatory diseases}

39 Multiple sclerosis (MS), 40 Meningeal irritation, 41 chronic meningitis, 42 paraneoplastic syndromes (including: 42a limbic encephalitis, including 42a-i paraneoplastic limbic encephalitis (PNLE), 42a-ii non-paraneoplastic limbic encephalitis (NPLE), 42b anti-NMDA receptor-mediated limbic encephalitis), 43 primary central nervous system vasculitis (PACNS)/primary centra nervous system angiitis (including: 43a neuro-Behçet, 43b systemic lupus erythematosus (SLE) 43c systemic vasculitides, 43d autoimmune connective tissue disorders, 43e polyarteritis, 43f nodosa, 43g Wegener's syndrome, 43h rheumatoid arthritis), 44 secondary central nervous system vasculitis, 45 neurosarcoidosis, 46 hepatic encephalopathy, 47 chronic obstructive pulmonary disease (COPD), 48 Bürger's disease, 49 Hashimoto's encephalopathy, 50 nonvasculitic autoimmune inflammatory meningoencephalitis, 51 celiac disease, 52 pancreatic encephalopathy, 53 antibasal ganglia antibodies (AGBA), 54 Sjögren syndrome, 55 cryoglobulinemia, 56 atrophic gastritis.

IV. Infectious diseases (including IVa Prion diseases)

57 Human immunodeficiency virus (HIV)/acquired immunodeficiency syndrome (AIDS) dementia complex, 58 prion diseases/transmissible spongiform encephalopathy (TSE) (including: 58a Creutzfeldt-Jakob disease (CJD), 58b new variant Creutzfeldt-Jacob disease (vCJD)/bovine spongiform encephalopathy (BSE), 58c Gerstmann-Sträussler-Scheinker syndrome, $58 \mathrm{~d}$ Kuru), 59 fata familial insomnia, 60 neurosyphilis (including: 60a general paresis of the insane, 60b luetic cerebrovascular disease), 61 cytomegalovirus encephalitis (including: 61a herpes simplex encephalitis, 61b herpes zoster), 62 Lyme disease, 63 immune thrombocytopenic purpura (ITP), 64 tuberculosis, 65 fungal infection, 66 epidemic encephalitis/encephalitis lethargica, 67 Whipple's disease, 68 infective endocarditis, 69 subacute sclerosing panencephalitis (SSPE), 70 neurocysticercosis, 71 progressive multifocal leukoencephalopathy, 72 toxoplasmosis.

Va. Toxic diseases/Vb. metabolic diseases/Vc. nutritional deficiencies

73 Alcohol-related dementias/alcohol abuse (including: 73a Wernicke syndrome, 73b Korsakoff syndrome, 73c Wernicke-Korsakoff syndrome, 73d presbyophrenia, 73e niacin deficiency/pellagra, 73f vitamin B12/thiamine deficiency, 73g Marchiafava-Bignami disease), 74 vitamin D deficiency, 75 vitamin E deficiency, 76 vitamin B9/folate deficiency, 77 drug-related dementias (including: 77a hashish, 77b barbiturates, 77c lithium, 77d interferon, 77e anti-cholinergic medication, 77f cocaine abuse, 77g methotrexate, 77h psychotropic drugs, 77i bromide, 77j phenothiazines, $77 \mathrm{k}$ valproate, $77 \mathrm{l}$ antiparkinson medication, $77 \mathrm{~m}$ ceftazidime toxicity), 78 heavy metal poisoning (including: $78 \mathrm{a}$ lead, $78 \mathrm{~b}$ mercury, $78 \mathrm{c}$ arsenic, $78 \mathrm{~d}$ manganese, $78 \mathrm{e}$ aluminium), 79 subacute combined degeneration of the cord/Lichtheim's disease/Putnam-Dana syndrome, 80 uraemia, 81 metabolic encephalopathy, 82 carbon monoxide poisoning, 83 hyperammonaemic encephalopathy/ citrullinemia, 84 renal failure and dialysis dementia, 85 citrullinemia, 86 obstructive sleep apnoea-hypnoea syndrome, 87 Wilson's disease, 88 porphyria, 89 electrolyte abnormalities, 90 Bismuth toxicity, 91 homocystinuria, 92 superficial siderosis, 93 mucopolysaccharidosis III, 94 ornithine transcobalamine deficiency, 95 ceftazidime toxicity, 96 abetalipoproteinemia, 97 galactosialidosis, 98 mannosidosis, 99 phenylketonuria (PKU), 100 hereditary spastic paraparesis (HSP/SPG4), 101 Lesch-Nyhan syndrome, 102 anti-voltage-gated potassium channel antibody syndrome (VGKC-Ab), 103 Leigh syndrome/subacute necrotizing encephalomeylopathy. 
Table 4

(Continued)

\section{Mitochondrial disorders}

104 Mitochondrial myopathy encephalopathy lactic acidosis and stroke (MELAS), 105 myoclonic epilepsy with ragged-red fibers (MERRF), 106 Kearns-Sayre syndrome, 107 DNA polymerase subunit gamma (POLG)-related disease.

VII. Lysosomal storage disorders

108 Tay-Sachs disease, 109 Gaucher's disease (including: 109a type 2, 109b type 3), 110 Niemann-Pick disease type C, 111 Fabry's disease, 112 neuronal ceroid lipofuscinosis (Kuf's disease or Batten disease), 113 GM 1 gangliosidosis, 114 GM2 gangliosidosis, 115 alpha mannosidosis, 116 adult Chediak-Higashi syndrome, 117 sialidosis (mucolipidosis I), 118 aspartylglucosaminuria. VIII. Leukodystrophies

119 X-linked adrenoleukodystrophy, 120 adrenoleukodystrophy, 121 metachromatic leukodystrophy, 122 Alexander's disease, 123 leukoencephalopathy with vanishing white matter, 124

Pelizaeus-Merzbacher disease, 125 adult polyglucosan body disease, 126 cerebrotendineous xanthomatosis, 127 pigmentary orthochromatic leukodystrophy (POLD), 128 hereditary

endotheliopathy with retinopathy nephropathy stroke (HERNS), 129 hereditary diffuse leukoencephalopathy with spheroids (HDLS), 130 Krabbe disease (globoid cell leukodystrophy), 13

polycystic lipomembranous leukodystrophy with sclerosing leukoencephalopathy (PLOSL/Nasu-Hakola disease), 132 Canavan disease.

IXa. Structural disorders/IXb. head injury/IXc. (para)neoplastic/metastatic

133 Cerebral tumors and abscess, 134 traumatic brain injury/head trauma, 135 normal pressure hydrocephalus, 136 subdural hematoma, 137 sequelae of cerebral laceration, 138 dural

arteriovenous fistula, 139 brain metastatic disease, 140 primary central nervous system lymphoma, 141 intravascular lymphoma, 142 lymphomatoid granulomatosis, 143 gliomatosis cerebri, 144

malignant melanoma, 145 chronic traumatic encephalopathy/dementia pugilistica, 146 hypereosinophilic syndrome.

X. Reversible disorders/treatable causes

147 Epileptic dementias (including: 147a temporal lobe epilepsy, 147b mesiotemporal lobe epilepsy), 148 iatrogenic disorders/adverse effects of prescribed medication (including: 148a post-irradiation lower motor neuron syndrome), 149 transient global amnesia (TGA)

XI. Endocrine disorders

150 Diabetes mellitus, 151 thyroid disease, 152 parathyroid disease (including: 152a hypoparathyroidism, 152b hyperparathyroidism), 153 hypothyroidism, 154 hyperthyroidism, 155 adrenal disease, 156 nonketonic hyperosmolar hyperglycaemia, 157 Cushing disease, 158 Addison disease, 159 pseudohypoparathyroidism, 160 hypopituitarism.

XII. Other (XIIa developmental disorders, XIIb psychiatric disorders)

161 Down's syndrome (trisomy 21), 162 depression, 163 anxiety, 164 conversion disorders, 165 mania, 166 schizophrenia.

XIII. Genetic disorders, XIV. hereditary disorders/heredo-degeneration, XV. (auto)immune, XVI. movement disorders, XVII. syphilitic dementias, XVIII. secondary dementias, XIX. dementia complicating chronic disorders, XX. multi-system failure, XXI. cerebral atrophy.

167 Immune-mediated diseases, 168 cerebellar atrophy (including: 168a olivopontocerebellar atrophy), 169 chronic hypoxia, 170 paroxysmal cardiac arrhythmia, 171 ureterosigmoidostomy, 172 acquired hepatocerebral degeneration. 
at a young age was explicitly mentioned, defined as "dementia plus syndromes" [4-6, 28, 32]. According to these authors, syndromes that cause cognitive impairment and show additional neurological or systemic features should be included as etiology as well, e.g., inclusion of diseases such as spinocerebellar ataxias and neurological features such as pyramidal signs are present in these syndromes [5]. Diseases and disorders mentioned as potential etiologies that do not conform to the customary criteria [48] include Down's syndrome or trisomy 21 [5, 29, 33, 34, 49], epilepsy [4-6, 25, 28, 43, 47, 50, 51], reversible or treatable causes [25, 28, 49, 50, 52, 53], depression $[32,47]$, and psychiatric disorders [47].

While no additional etiologies of dementia at a young age were found in the category 2 literature, they provided new insights into the reasons for the inclusion and exclusion of various etiologies. In the methodology section of six publications, a total of 36 etiologies were explicitly mentioned as exclusion criteria, such as cognitive impairment secondary to other pathologies (e.g., psychiatric disorders or neurological disorders without a progressive course) specialized care organized by other services, or not fitting with internationally accepted criteria for diagnosing dementia [54-57]. Disorders and diseases mentioned as exclusion criteria most often included depression or psychiatric disorders [54, 56-58], Huntington's chorea $[54,55]$, developmental disorders or Down's syndrome [55, 58], alcohol abuse or Korsakoff's syndrome [56, 59], epilepsy [54-56, 59], human immunodeficiency virus [55], and traumatic brain injury [54-56, 59].

In addition to the large number of unique etiologies of dementia at a young age, 27 overarching categories were identified. The description of etiologies in categories was either used to give a structured overview of the large number of etiologies [41] or as a hierarchical way of organizing etiologies [38]. The categories mentioned most often were toxic and metabolic disorders (18 publications), infectious diseases (15), primary neurodegenerative disorders (14), cerebrovascular dementias (13), and structural and paraneoplastic disorders (11). No additional categories were identified in the sample of category 2 literature.

\section{DISCUSSION}

To our knowledge, this is the first review presenting both key aspects concerning terminology and definition of dementia at a young age as well as an inclusive list of etiologies. In recent years, there seems to be relative consensus on the term young-onset dementia, which has become the most commonly used term to refer to young people living with dementia. The age of 65 years at symptom onset was used most widely to distinguish this group from people living with dementia in old age. Furthermore, over 251 different etiologies were identified that might cause dementia at a young age. Most etiologies and overarching categories were mentioned frequently, and the remaining few etiologies and categories could be considered controversial. However, large heterogeneity and several controversies were observed regarding age-related criteria and the inclusion of various etiologies.

The fact that the term young-onset dementia was used most often in the last two decades is not only a historical evolution, but content-driven. Most frequently given reasons for the use of this particular term, rather than presenile dementia, were of biological origin, as young-onset dementia incorporates typical etiologies found in old-age dementia, rare etiologies found in middle-aged dementia and late-onset forms of childhood neurodegenerative disorders. Notably, many authors did not provide argumentation to explain the use of a specific term. Additionally, the term early onset dementia, which was the most commonly used until 2013, is still used by some authors $[33,60]$. This might be explained by the fact that some authors reserve this particular term to refer to young people living with typical old-age causes of dementia, while others used this term to refer to people aged between 45 and 65 as comparison with people below age 45 .

The age of 65 years at symptom onset was most frequently used as the cut-off age and criterion to differentiate from late-onset dementia. The most commonly used argument for the use of this particular cut-off age and the use of age at symptom onset instead of age at diagnosis was retirement age. Some authors mentioned a biological argument for this cutoff age, albeit arbitrary, as rare causes often occur below the age of 65 years.

Furthermore, in a minority of the literature, a lower age limit to distinguish developmental disorders was observed. Adoption of a lower age limit clearly distinguishes dementia from developmental disorders. On the other hand, the prevalence of dementia becomes rarer at younger ages [29]. Together with the large variety in suggested age ranges found in the literature, i.e., between 16 and 45 years, this raises the 
question of whether such a criterion should be set and at what age. The few publications mentioning a lower age limit provided little argument for this criterion. The decisions for specific ages were often based on own clinical experiences rather than on biological or psychosocial theories, according to descriptions such as 'from early adulthood' [28] or 'have occurred in older and much younger patients' [42]. The use of different upper and lower age limits in the various sources resulted in differences in patient groups, which make these groups difficult to compare with one-another

In seven publications, the authors also proposed a subdivision into two age groups because dementia is more often caused by rare diseases and disorders, especially in people below the age of 45 . Consequently, from a care perspective, such individuals may likely experience very specific problems, such as diagnostic delays, psychosocial problems, and heredity, which are more common. This underlines the importance of differentiating between the youngest young and people aged between 45 and 65 years.

There is a known large heterogeneity in causes of dementia in younger individuals [5]. However, some etiologies and categories could be considered controversial because clustering of etiologies can be done in several ways and might result in overlapping categories. Furthermore, diseases and disorders do not meet all Diagnostic and Statistical Manual of Mental Disorders (DSM-V) criteria for neurocognitive disorders [48]. In fact, several conditions were mentioned as etiologies by some authors but mentioned as exclusion criteria by others. These etiologies included Down's syndrome and other (neuro)developmental disorders, epilepsy and other reversible or treatable diseases, depression and other psychiatric disorders, Huntington's chorea, alcohol dementia, Korsakoff's syndrome, human immunodeficiency virus, and traumatic brain injury. In addition to these identified controversial etiologies, others might yet be determined. For instance, multiple system atrophy can cause cognitive decline in more advanced stages but is primarily considered a movement disorder, and most people living with multiple system atrophy die before the onset of severe cognitive symptoms [61]. Last, only few authors explicated the medical choice for inclusion or exclusion. In most literature including for example Down's syndrome or Huntington's chorea, it remains unclear whether these etiologies in itself caused dementia or whether these disorders were associated with dementia.

\section{Implications}

In this review, we identified many elements considered in the nomenclature and definition of dementia at a young age. The largest research gap is the explication of underlying considerations, such as from what perspective (e.g., psychosocial or biological) agerelated criteria and included etiologies were defined in the various publications. The predominantly interchangeable use of terms and definitions has four major consequences. First, whether a biological or psychosocial perspective was used influenced the choice in terminology. The term early-onset dementia is reserved by some authors to refer to young people living with dementia caused by typical old-age etiologies aged below 65 years-based on retirement and thereby chosen from a psychosocial perspective. Others reserved early-onset dementia to refer to people living with dementia aged below 65 years but above 45 years-because there are etiological differences compared to the group of people below 45 years living with dementia and thereby chosen from a biological perspective. Second, the choice between the age at symptom onset or at diagnosis as a cut-off criterion has significant consequences for determining the number of people living with dementia considered young, as it can take up to 4.7 years between the onset of first symptoms and final diagnosis of the type of dementia [62]. Next to the amount of people that might be referred to post-diagnostic services, there also needs to be policy developed for these services regarding inclusion or exclusion of people with age of onset somewhere ambiguous around the age of 65 years. Third, although awareness of young people living with dementia is increasing, dementia services remain primarily focused on the needs of older people living with dementia [18], and a subdivision could scatter the awareness and the amount of age-related services. On the other hand, such a subdivision could be beneficial from a biological or research perspective to identify specific disease mechanisms in dementias with onset at a young age. Last, the inclusion of all etiologies found in this review as possible causes would significantly increase the determination of the number of young people living with dementia at a young age, which would have an effect on the number of post-diagnostic care facilities needed for this specific group of patients. On the one hand, it seems reasonable that people living with dementia at a young age and their caregivers might have similar needs regardless of the etiology, resulting in, for instance, multiple system atrophy as etiology. On the other 
hand, specialized care for etiologies and categories, such as Down's syndrome and other developmental disorders or Huntington's chorea, are often already embedded in other care systems and are financed differently. New insights might add to the discussion; for instance, amyotrophic lateral sclerosis is linked to frontotemporal lobar degeneration and might be a potential etiology [63].

\section{Strengths and limitations}

There are several strengths to this integrative review. A comprehensive search strategy was used in which both empirical literature and grey literature were combined. Additionally, this study was conducted using a rigorous methodology encompassing both a structured approach and a tailored quality appraisal [20]. Despite these strengths, there are some limitations that must be addressed. First, possible publication bias could be present, as only published literature was selected. Possible internal documents or publications otherwise not published were not included. This might have resulted in an underrepresentation of studies, especially in low-income countries [64], causing certain aspects to be missed, such as potential region-specific diseases. Additionally, because we only included sources written in English or Dutch, it is likely that some national policy documents containing specific local age-related criteria were missed or potential region-specific diseases. As a third limitation, albeit not of methodological nature, we found that only a few publications included substantiated arguments for their choices. Many definitions were implicitly chosen, which did not allow us to gather information on whether these choices were based on opinions or specific empirical arguments.

\section{CONCLUSIONS}

Throughout history, several different terms to refer to young people living with dementia have been used interchangeably, and a clear definition in terms of ages and etiologies is lacking. This causes ambiguity, which complicates comparing research and determining the number of young people living with dementia, representing a barrier to improving post-diagnostic support. The explication of an operational definition used in each study is recommended for future research. The current study identified three major key points that might help clarify these abovementioned issues: a commonly used term, a frequently used cut-off age and a comprehensive list of etiologies of dementia at a young age. A consensus study could help reach international agreement concerning the terminology and definition and should include space to elaborate, which in turn could aid in the clarification of this specific group of people living with dementia. A clear definition is beneficial for conducting research and for clinicians, for example to develop policies concerning the organization of ageappropriate post-diagnostic services.

\section{ACKNOWLEDGMENTS}

Funding for this article was provided by the Gieskes-Strijbis Fund, Alzheimer Netherlands, the Dutch Young-onset Dementia Knowledge Center and Florence Care Group in The Netherlands. We thank the PRECODE project team for their suggestions and collaboration.

Authors' disclosures available online (https:// www.j-alz.com/manuscript-disclosures/21-0458r1).

\section{SUPPLEMENTARY MATERIAL}

The supplementary material is available in the electronic version of this article: https://dx.doi.org/ 10.3233/JAD-210458.

\section{REFERENCES}

[1] World Health Organization, Dementia, https://www.who. int/news-room/fact-sheets/detail/dementia,

[2] Graeber MB, Kosel S, Egensperger R, Banati RB, Muller U, Bise K, Hoff P, Moller HJ, Fujisawa K, Mehraein P (1997) Rediscovery of the case described by Alois Alzheimer in 1911: Historical, histological and molecular genetic analysis. Neurogenetics 1, 73-80.

[3] Miyoshi K (2009) What is 'early onset dementia'? Psychogeriatrics 9, 67-72.

[4] Kuruppu DK, Matthews BR (2013) Young-onset dementia. Semin Neurol 33, 365-385.

[5] Sampson EL, Warren JD, Rossor MN (2004) Young onset dementia. Postgrad Med J 80, 125-139.

[6] Rossor MN, Fox NC, Mummery CJ, Schott JM, Warren JD (2010) The diagnosis of young-onset dementia. Lancet Neurol 9, 793-806.

[7] van der Vlies AE, Koedam EL, Pijnenburg YA, Twisk JW, Scheltens P, van der Flier WM (2009) Most rapid cognitive decline in APOE epsilon4 negative Alzheimer's disease with early onset. Psychol Med 39, 1907-1911.

[8] Gerritsen AAJ, Bakker C, Verhey FRJ, Bor H, Pijnenburg YAL, de Vugt ME, Koopmans R (2018) The progression of dementia and cognitive decline in a Dutch 2-year cohort study of people with young-onset dementia. J Alzheimers Dis 63, 343-351.

[9] Mendez MF (2017) Early-onset Alzheimer disease. Neurol Clin 35, 263-281. 
[10] Koedam EL, Lauffer V, van der Vlies AE, van der Flier WM, Scheltens P, Pijnenburg YA (2010) Early-versus late-onset Alzheimer's disease: More than age alone. J Alzheimers Dis 19, 1401-1408.

[11] van Vliet D, de Vugt ME, Kohler S, Aalten P, Bakker C, Pijnenburg YA, Vernooij-Dassen MJ, Koopmans RT, Verhey FR (2013) Awareness and its association with affective symptoms in young-onset and late-onset Alzheimer disease: A prospective study. Alzheimer Dis Assoc Disord 27, 265-271.

[12] Neary D, Snowden JS, Gustafson L, Passant U, Stuss D, Black S, Freedman M, Kertesz A, Robert PH, Albert M, Boone K, Miller BL, Cummings J, Benson DF (1998) Frontotemporal lobar degeneration: A consensus on clinical diagnostic criteria. Neurology 51, 1546-1554.

[13] van Vliet D, de Vugt ME, Bakker C, Pijnenburg YA, Vernooij-Dassen MJ, Koopmans RT, Verhey FR (2013) Time to diagnosis in young-onset dementia as compared with late-onset dementia. Psychol Med 43, 423-432.

[14] Millenaar JK, Bakker C, Koopmans RT, Verhey FR, Kurz A, de Vugt ME (2016) The care needs and experiences with the use of services of people with young-onset dementia and their caregivers: A systematic review. Int J Geriatr Psychiatry 31, 1261-1276.

[15] van Vliet D, Persoon A, Bakker C, Koopmans R, de Vugt ME, Bielderman A, Gerritsen DL (2017) Feeling useful and engaged in daily life: Exploring the experiences of people with young-onset dementia. Int Psychogeriatr 29, 1889-1898.

[16] Kandiah N, Wang V, Lin X, Nyu MM, Lim L, Ng A, Hameed S, Wee HL (2016) Cost related to dementia in the young and the impact of etiological subtype on cost. $J$ Alzheimers Dis 49, 277-285.

[17] Werner P, Stein-Shvachman I, Korczyn AD (2009) Early onset dementia: Clinical and social aspects. Int Psychogeriatr 21, 631-636.

[18] Carter JE, Oyebode JR, Koopmans R (2018) Young-onset dementia and the need for specialist care: A national and international perspective. Aging Ment Health 22, 468-473.

[19] Farrer LA, Cupples LA, Haines JL, Hyman B, Kukull WA, Mayeux R, Myers RH, Pericak-Vance MA, Risch N, van Duijn CM (1997) Effects of age, sex, and ethnicity on the association between apolipoprotein E genotype and Alzheimer disease. A meta-analysis. APOE and Alzheimer Disease Meta Analysis Consortium. JAMA 278, 1349-1356.

[20] Whittemore R, Knafl K (2005) The integrative review: Updated methodology. J Adv Nurs 52, 546-553.

[21] Cooper HM (1998) Synthesizing Research: A Guide for Literature Reviews, Sage Publications, Thousand Oaks, CA.

[22] Ouzzani M, Hammady H, Fedorowicz Z, Elmagarmid A (2016) Rayyan-a web and mobile app for systematic reviews. Syst Rev 5, 210.

[23] Critical Appraisal Skills Programme (2018), Oxford, UK.

[24] Tyndall J, The AACODS checklist, Dspace Flinders, https://dspace.flinders.edu.au/jspui/bitstream/2328/3326/ AACODS_Checklist.pdf,

[25] Davies RR, Doran M, Larner AJ (2011) Diagnosis and management of early-onset dementia. Prog Neurol Psychiatry 15, 12-16.

[26] Greicius MD, Geschwind MD, Miller BL (2002) Presenile dementia syndromes: An update on taxonomy and diagnosis. J Neurol Neurosurg Psychiatry 72, 691-700.
[27] Fujihara S, Brucki SM, Rocha MS, Carvalho AA, Piccolo AC (2004) Prevalence of presenile dementia in a tertiary outpatient clinic. Arq Neuropsiquiatr 62, 592-595.

[28] Masellis M, Sherborn K, Neto P, Sadovnick DA, Hsiung GY, Black SE, Prasad S, Williams M, Gauthier S (2013) Early-onset dementias: Diagnostic and etiological considerations. Alzheimers Res Ther 5, S7.

[29] Harvey RJ (1998) The Impact of Young Onset Dementia. A study of the epidemiology, clinical features, caregiving and health economics of dementia in younger people. Thesis. Imperial College School of Medicine.

[30] Withall A (2013) The challenges of service provision in younger-onset dementia. JAm MedDirAssoc 14, 230-232.

[31] Vafeas C, Jacob E, Jacob A (2020) A younger onset dementia toolkit: Innovative practice. Dementia (London) 19, 1299-1307.

[32] Draper B, Withall A (2016) Young onset dementia. Intern Med J 46, 779-786.

[33] NHMRC National Institute for Dementia Research (2020) Australia dementia forum, abstracts. Accelerating Research. Enhancing Collaboration. Creating Change, Adelaide Convention Centre.

[34] Hayo H (2015) Diagnosis and support for younger people with dementia. Nurs Stand 29, 36-40.

[35] Koopmans R, Rosness T (2014) Young onset dementiawhat does the name imply? Int Psychogeriatr 26, 1931-1933.

[36] Tonkonogy J, Moak GS (1988) Alois Alzheimer on presenile dementia. J Geriatr Psychiatry Neurol 1, 199-206.

[37] Dixon BK (2007) Early-onset Alzheimer's gets name change. Caring Ages 8, 14.

[38] Goldman JS (2015) Genetic testing and counseling in the diagnosis and management of young-onset dementias. Psychiatr Clin North Am 38, 295-308.

[39] Risien Russel JS (1933) Discussion on the mental and physical symptoms of the presenile dementias: Joint discussion no. 5. Proc R Soc Med 26, 1077-1091.

[40] Kelley BJ, Boeve BF, Josephs KA (2008) Young-onset dementia: Demographic and etiologic characteristics of 235 patients. Arch Neurol 65, 1502-1508.

[41] Doran M (1997) Diagnosis of presenile dementia. Br J Hosp Med 58, 105-110.

[42] Gibson J (1960) Presenile dementia. Can Nurse 56, 356358.

[43] Goodall E (1938) The presenile dementias: Symptomatology, pathology, and differential diagnosis: (section of psychiatry and section of neurology), joint discussion no. 6. Proc R Soc Med 31, 1443-1454.

[44] Moffie D (1953) Presenile dementia. Folia Psychiatr Neurol Neurochir Neerl 56, 360-374.

[45] Pinhorn A (1993) Presenile dementia. Dementia classification misleading. BMJ 306, 1343-1344.

[46] Harvey RJ, Skelton-Robinson M, Rossor MN (2003) The prevalence and causes of dementia in people under the age of 65 years. J Neurol Neurosurg Psychiatry 74, 1206-1209.

[47] Ridha B, Josephs KA (2006) Young-onset dementia: A practical approach to diagnosis. Neurologist 12, 2-13.

[48] American Psychiatric Association (2013) Diagnostic and statistical manual of mental disorders. Washington, DC.

[49] Fadil H, Borazanci A, Ait Ben Haddou E, Yahyaoui M, Korniychuk E, Jaffe SL, Minagar A (2009) Early onset dementia. Int Rev Neurobiol 84, 245-262.

[50] Ahmed S, Baker I, Butler CR (2016) Diagnosing young onset dementia can be challenging. Practitioner 260, 1117,2 . 
[51] Liebson E, Rauch P, Graff S, Folstein M (2005) Earlyonset dementia: Diagnostic considerations and implications for families. Harv Rev Psychiatry 13, 102-111.

[52] Charlton MH (1975) Presenile dementia. N Y State J Med 75, 1493-1495.

[53] Sitek EJ, Barczak A, Harciarek M (2015) Neuropsychological assessment and differential diagnosis in young-onset dementias. Psychiatr Clin North Am 38, 265-279.

[54] Allen H, Baldwin B (1995) The referral, investigation and diagnosis of presenile dementia: Two services compared. Int J Geriatr Psychiatry 10, 185-190.

[55] Appelhof B, Bakker C, de Vugt ME, van Duinen-van den IJssel JCL, Zwijsen SA, Smalbrugge M, Teerenstra S, Verhey FRJ, Zuidema SU, Koopmans RTCM (2019) Effects of a multidisciplinary intervention on the presence of neuropsychiatric symptoms and psychotropic drug use in nursing home residents withyoung-onset dementia: Behavior And Evolution Of Young-Onset Dementia Part 2 (BEYOND-II) Study. Am J Geriatr Psychiatry 27, 581-589.

[56] Jefferies K, Agrawal N (2009) Early-onset dementia. Adv Psychiatr Treat 15, 380-388.

[57] Nordstrom P, Nordstrom A, Eriksson M, Wahlund LO, Gustafson Y (2013) Risk factors in late adolescence for young-onset dementia in men: A nationwide cohort study. JAMA Intern Med 173, 1612-1618.

[58] Ikejima C, Yasuno F, Mizukami K, Sasaki M, Tanimukai S, Asada T (2009) Prevalence and causes of early-onset dementia in Japan: A population-based study. Stroke 40, 2709-2714.

[59] Ron MA, Toone BK, Garralda ME, Lishman WA (1979) Diagnostic accuracy in presenile dementia. Br J Psychiatry 134, 161-168.

[60] Wawrziczny E, Berna G, Ducharme F, Kergoat MJ, Pasquier F, Antoine P (2018) Characteristics of the spouse caregiving experience: Comparison between early- and late-onset dementia. Aging Ment Health 22, 1207-1215.

[61] Stankovic I, Krismer F, Jesic A, Antonini A, Benke T, Brown RG, Burn DJ, Holton JL, Kaufmann H, Kostic VS, Ling H, Meissner WG, Poewe W, Semnic M, Seppi K, Takeda A, Weintraub D, Wenning GK; Movement Disorders Society MSA Study Group (2014) Cognitive impairment in multiple system atrophy: A position statement by the Neuropsychology Task Force of the MDS Multiple System Atrophy (MODIMSA) study group. Mov Disord 29, 857-867.

[62] Draper B, Cations M, White F, Trollor J, Loy C, Brodaty H, Sachdev P, Gonski P, Demirkol A, Cumming RG, Withall A (2016) Time to diagnosis in young-onset dementia and its determinants: The INSPIRED study. Int J Geriatr Psychiatry 31, 1217-1224.

[63] Seelaar H, Rohrer JD, Pijnenburg YA, Fox NC, van Swieten JC (2011) Clinical, genetic and pathological heterogeneity of frontotemporal dementia: A review. J Neurol Neurosurg Psychiatry 82, 476-486.

[64] Prince M (2000) Methodological issues for populationbased research into dementia in developing countries. A position paper from the 10/66 Dementia Research Group. Int J Geriatr Psychiatry 15, 21-30.

[65] Coblentz JM, Mattis S, Zingesser LH, Kasoff SS, Wisniewski HM, Katzman R (1973) Presenile dementia. Clinical aspects and evaluation of cerebrospinal fluid dynamics. Arch Neurol 29, 299-308.
[66] Cowie V (1981) Presenile dementia. Old before your time. Nurs Mirror 152, 44-45.

[67] Forbes K (2005) A multi-disciplinary approach to diagnosis and assessment in early-onset dementia. Cortex $\mathbf{4 1}$, 90-95.

[68] Giannakopoulos P, Hof PR, Savioz A, Guimon J, Antonarakis SE, Bouras C (1996) Early-onset dementias: Clinical, neuropathological and genetic characteristics. Acta Neuropathol 91, 451-465.

[69] Laino C (2005) Early-onset dementia is often due to preventable causes - and PET helps diagnosis. Neurol Today 5, 47-48.

[70] Levine DA (2013) Young-onset dementia: Unanswered questions and unmet needs. JAMA Intern Med 173, 1619-1620.

[71] Panegyres PK, Davies SR, Connor CF (2000) Early-onset dementia. Med J Aust 173, 279-280.

[72] Poser CM (1975) The presenile dementias. JAMA 233, 81-84.

[73] (2016) Spotting early onset dementia. Nurs Stand 31, 66.

[74] Andrew C, Phillipson L, Sheridan L (2019) What is the impact of dementia on occupational competence, occupational participation and occupational identity for people who experience onset of symptoms while in paid employment? A scoping review. Aust Occup Ther J 66, 130-144.

[75] Armari E, Jarmolowicz A, Panegyres PK (2013) The needs of patients with early onset dementia. Am J Alzheimers Dis Other Demen 28, 42-46.

[76] Arvanitakis Z, Shah RC, Bennett DA (2019) Diagnosis and management of dementia: Review. JAMA 322, 1589-1599.

[77] Balasa M, Sanchez-Valle R, Antonell A, Bosch B, Olives J, Rami L, Castellvi M, Molinuevo JL, Llado A (2014) Usefulness of biomarkers in the diagnosis and prognosis of early-onset cognitive impairment. J Alzheimers Dis 40, 919-927.

[78] Belhouane I, Djebara MB, Hizem Y, Gargouri A, Gouider R (2012) Early onset dementia: A Tunisian cohort. Neurology. Conference: 64th American Academy of Neurology Annual Meeting. New Orleans, LA United States. Conference Publication.

[79] Cations M, Withall A, Draper B (2019) Modifiable risk factors for young onset dementia. Curr Opin Psychiatry 32, 138-143.

[80] Dale MC (2000) Need for neuropathological studies in pre-senile dementia. Br J Psychiatry 177, 565-566.

[81] Devineni B, Onyike CU (2015) Young-onset dementia epidemiology applied to neuropsychiatry practice. Psychiatr Clin North Am 38, 233-248.

[82] Diehl-Schmid J, Hartmann J, Rossmeier C, Riedl L, Forstl H, Egert-Schwender S, Kehl V, Schneider-Schelte H, Jox RJ (2018) IssuEs in Palliative care for people in advanced and terminal stages of Young-onset and LateOnset dementia in GErmany (EPYLOGE): The study protocol. BMC Psychiatry 18, 271.

[83] Dumitru MM, Chirita V, Chirita R (2014) P3-171: Characteristics of early onset dementia in a hospital setting from Romania. Alzheimers Dement 10, P691.

[84] Filipovic SR, Kostic VS (1995) Utility of auditory P300 in detection of presenile dementia. J Neurol Sci 131, 150-155.

[85] Garre-Olmo J, Genis Batlle D, del Mar Fernandez M, Marquez Daniel F, de Eugenio Huelamo R, Casadevall T, Turbau Recio J, Turon Estrada A, Lopez-Pousa S; Registry of Dementia of Girona Study Group (ReDeGi Study Group) (2010) Incidence and subtypes of early-onset 
dementia in a geographically defined general population. Neurology 75, 1249-1255.

[86] Giebel C, Eastham C, Cannon J, Wilson J, Wilson J, Pearson A (2020) Evaluating a young-onset dementia service from two sides of the coin: Staff and service user perspectives. BMC Health Serv Res 20, 187.

[87] Gordon EB (1968) Serial EEG studies in presenile dementia. Br J Psychiatry 114, 779-780.

[88] Gottfries CG, Gottfries I, Roos BE (1969) Homovanillic acid and 5-hydroxyindoleacetic acid in the cerebrospinal fluid of patients with senile dementia, presenile dementia and parkinsonism. J Neurochem 16, 1341-1345.

[89] Guermani M, Robbana L, Moula O, Bouasker A, Ghachem R (2013) Early onset dementia: Socio-demographic and clinical characteristics. Eur Psychiatry 28(S1), 1.

[90] Gustafson L, Nilsson L (1982) Differential diagnosis of presenile dementia on clinical grounds. Acta Psychiatr Scand 65, 194-209.

[91] Henley SMD, Crutch S, Mummery CJ, Caine D (2014) P3-270: A novel peri-diagnostic counseling service for patients and families with rare, inherited, or young-onset dementias. Alzheimers Dement 10, P730.

[92] Hutchinson K, Roberts C, Kurrle S, Daly M (2016) The emotional well-being of young people having a parent with younger onset dementia. Dementia (London) 15, 609-628.

[93] Hvidsten L, Engedal K, Selbaek G, Wyller TB, Benth JS, Kersten H (2019) Quality of life in people with young-onset dementia: A Nordic two-year observational multicenter study. J Alzheimers Dis 67, 197-210.

[94] Johannessen A, Moller A, Haugen PK, Biong S (2014) A shifting sense of being: A secondary analysis and comparison of two qualitative studies on young-onset dementia. Int J Qual Stud Health Well-being 9, 24756.

[95] Kaszniak AW, Fox J, Gandell DL, Garron DC, Huckman MS, Ramsey RG (1978) Predictors of mortality in presenile and senile dementia. Ann Neurol 3, 246-252.

[96] Kilty C, Boland P, Goodwin J, de Róiste Á (2019) Caring for people with young onset dementia: An interpretative phenomenological analysis of family caregivers' experiences. J Psychosoc Nurs Ment Health Serv 57, 37-44.

[97] Koopmans RT, van der Steen JT, Bakker C (2015) Palliative care in people with Young-Onset Dementia (YOD): An undiscovered area! J Am Med Dir Assoc 16, 1008-1009.

[98] Kurz A, Bakker C, Bohm M, Diehl-Schmid J, Dubois B, Ferreira C, Gage H, Graff C, Hergueta T, Jansen S, Jones B, Komar A, de Mendonca A, Metcalfe A, Milecka K,
Millenaar J, Orrung Wallin A, Oyebode J, SchneiderSchelte H, Saxl S, de Vugt M (2016) RHAPSODY Internet-based support for caregivers of people with young onset dementia: Program design and methods of a pilot study. Int Psychogeriatr 28, 2091-2099.

[99] Liddell D (1958) Investigations of EEG findings in presenile dementia. J Neurol Neurosurg Psychiatry 21, 173-176.

[100] Maiovis P, Ioannidis P, Konstantinopoulou E, Karacostas D (2015) Early onset degenerative dementias: Demographic characteristics and etiologic classification in a tertiary referral center. Acta Neurol Belg 115, 27-31.

[101] McMurtray A, Clark DG, Christine D, Mendez MF (2006) Early-onset dementia: Frequency and causes compared to late-onset dementia. Dement Geriatr Cogn Disord 21, 59-64.

[102] Phuapradit P, Phillips M, Lees AJ, Stern GM (1978) Bromocriptine in presenile dementia. Br Med J 1, 10521053.

[103] Rizzo PA, Albani G, Cicardi C, Spadaro M, Morocutti C (1984) Effects of distraction on the contingent negative variation in presenile dementia and normal subjects. Neuropsychobiology 12, 112-114.

[104] Rogers BS, Lippa CF (2012) A clinical approach to earlyonset inheritable dementia. Am J Alzheimers Dis Other Demen 27, 154-161.

[105] Sansoni J, Duncan C, Grootemaat P, Capell J, Samsa P, Westera A (2016) Younger Onset Dementia. Am J Alzheimers Dis Other Demen 31, 693-705.

[106] Smith WT, Turner E, Sim M (1966) Cerebral biopsy in the investigation of presenile dementia. II. Pathological aspects. Br J Psychiatry 112, 127-133.

[107] Spreadbury J, Kipps C (2019) Measuring younger onset dementia: A comprehensive literature search of the quantitative psychosocial research. Dementia (London) $\mathbf{1 8}$ 135-156.

[108] Torvik A (1970) Aspects of the pathology of presenile dementia. Acta Neurol Scand 46, 19-31.

[109] Vieira RT, Caixeta L, Machado S, Silva AC, Nardi AE, Arias-Carrion O, Carta MG (2013) Epidemiology of earlyonset dementia: A review of the literature. Clin Pract Epidemiol Ment Health 9, 88-95.

[110] Woodburn K, Johnstone E (1999) Measuring the decline of a population of people with early-onset dementia in Lothian, Scotland. Int J Geriatr Psychiatry 14, 355-361.

[111] Wright CE, Harding GF, Orwin A (1984) Presenile dementia-the use of the flash and pattern VEP in diagnosis. Electroencephalogr Clin Neurophysiol 57, 405-415. 